\title{
Exploring the Diversity and Regulation of Apocarotenoid Metabolic Pathways in Plants
}

\author{
Xiongjie Zheng, Yu Yang and Salim Al-Babili* \\ The BioActives Lab, Center for Desert Agriculture (CDA), Biological and Environment Science and Engineering (BESE), King \\ Abdullah University of Science and Technology, Thuwal, Saudi Arabia
}

\section{OPEN ACCESS}

Edited by:

Lourdes Gómez-Gómez,

University of Castilla-La

Mancha, Spain

Reviewed by:

Maria Jesus Rodrigo, Institute of Agrochemistry and Food

Technology, Spanish National

Research Council (CSIC), Spain

Akemi Ohmiya,

National Agriculture and Food

Research Organization (NARO),

Japan

${ }^{*}$ Correspondence:

Salim Al-Babill

salim.babili@kaust.edu.sa

Specialty section:

This article was submitted to

Plant Metabolism and

Chemodiversity,

a section of the journal

Frontiers in Plant Science

Received: 30 September 2021

Accepted: 17 November 2021

Published: 10 December 2021

Citation:

Zheng $X$, Yang $Y$ and

Al-Babili S (2021) Exploring the

Diversity and Regulation of Apocarotenoid Metabolic Pathways

in Plants.

Front. Plant Sci. 12:787049.

doi: 10.3389/fpls.2021.787049
In plants, carotenoids are subjected to enzyme-catalyzed oxidative cleavage reactions as well as to non-enzymatic degradation processes, which produce various carbonyl products called apocarotenoids. These conversions control carotenoid content in different tissues and give rise to apocarotenoid hormones and signaling molecules, which play important roles in plant growth and development, response to environmental stimuli, and in interactions with surrounding organisms. In addition, carotenoid cleavage gives rise to apocarotenoid pigments and volatiles that contribute to the color and flavor of many flowers and several fruits. Some apocarotenoid pigments, such as crocins and bixin, are widely utilized as colorants and additives in food and cosmetic industry and also have health-promoting properties. Considering the importance of this class of metabolites, investigation of apocarotenoid diversity and regulation has increasingly attracted the attention of plant biologists. Here, we provide an update on the plant apocarotenoid biosynthetic pathway, especially highlighting the diversity of the enzyme carotenoid cleavage dioxygenase 4 (CCD4) from different plant species with respect to substrate specificity and regioselectivity, which contribute to the formation of diverse apocarotenoid volatiles and pigments. In addition, we summarize the regulation of apocarotenoid metabolic pathway at transcriptional, post-translational, and epigenetic levels. Finally, we describe inter- and intraspecies variation in apocarotenoid production observed in many important horticulture crops and depict recent progress in elucidating the genetic basis of the natural variation in the composition and amount of apocarotenoids. We propose that the illustration of biochemical, genetic, and evolutionary background of apocarotenoid diversity would not only accelerate the discovery of unknown biosynthetic and regulatory genes of bioactive apocarotenoids but also enable the identification of genetic variation of causal genes for marker-assisted improvement of aroma and color of fruits and vegetables and CRISPR-based next-generation metabolic engineering of highvalue apocarotenoids.

Keywords: carotenoids, apocarotenoids, pigments, volatiles, natural variation, molecular regulation 


\section{INTRODUCTION}

Carotenoids are lipophilic isoprenoid molecules consisting of a polyene backbone that generally contains 3-11 conjugated double bounds. They can be synthesized de novo by plants, algae, photosynthetic bacteria, and many nonphotosynthetic microorganisms (Hirschberg, 2001; Fraser and Bramley, 2004; DellaPenna and Pogson, 2006; Moise et al., 2014; RodriguezConcepcion et al., 2018; Zheng et al., 2020a). Humans cannot build carotenoids de novo, but need them as important antioxidants and as essential source of provitamin A, particularly if animal-derived food is not available (Fraser and Bramley, 2004; DellaPenna and Pogson, 2006; Giuliano, 2014; Nisar et al., 2015; Zheng et al., 2020b).

In many crops and other plant species, carotenoids confer their vivid yellow to red colors to fruits, flowers, tubers, and seeds (Yuan et al., 2015; Giuliano, 2017), which attract animal pollinators and distributors and are important feature for consumers, which indicate the quality of plant products and, hence, promote their marketability. More importantly, carotenoids are essential for plant photosynthesis, as they protect the photosynthetic apparatus from photooxidative damage and contribute to light-harvesting (Hashimoto et al., 2016; RodriguezConcepcion et al., 2018; Moreno et al., 2021). The conjugated double bonds make carotenoids themselves susceptible to photooxidation and other unspecific oxidative breakdown processes, such as lipoxygenase mediated co-oxidation, as well as to targeted, enzyme-catalyzed cleavage of certain double bonds (Zheng et al., 2020a; Moreno et al., 2021). The term "apocarotenoid" is used to define the cleavage products arising from these processes. The large number of double bonds in carotenoid backbone and of different carotenoids leads to a plentitude of apocarotenoids with various physico-chemical properties and biological functions. The compound family of apocarotenoids includes precursors of important phytohormones, that is, abscisic acid (ABA) and strigolactones (SLs), signaling molecules involved in plant growth, development, and resistance against pathogens and herbivores (Jia et al., 2018; Felemban et al., 2019; Watkins and Pogson, 2020; Wang et al., 2020b), as well as apocarotenoid pigments and volatiles responsible for color and aroma of fruits, flowers, or vegetables (Liang et al., 2021; Zheng et al., 2021).

Plant apocarotenoids and their derivatives have also important functions in promoting human health (Rodriguez-Concepcion et al., 2018). Humans contain two carotenoid cleavage enzymes, i.e., $\beta$-carotene cleavage oxygenase 1 and 2 (BCO1 and 2), which convert some carotenoids into a restricted set of apocarotenoids, including vitamin A (Harrison, 2005; Von Lintig, 2010); however, they cannot produce many health-promoting apocarotenoids, such as crocins and safranal (Frusciante et al., 2014; Ahrazem et al., 2016b). Given the importance of apocarotenoids for plants and humans, numerous studies have been performed on investigating the biological functions of apocarotenoids, elucidating their biosynthetic pathways and the regulation of their formation. Naturally occurring inter- or intraspecies apocarotenoid variation was observed in some important crops, such as citrus, tomato, and Capsicum species (Gao et al., 2019; Zheng et al., 2019; Zoccali et al., 2021), which would help to decipher apocarotenoid biosynthesis and its regulation, and genetic control of apocarotenoid diversity. Nowadays, the advancements in global profiling strategies, such as genomics, transcriptomics, and metabolomics, and multiomics approaches have been very helpful for investigating the variation of apocarotenoid levels as well as the underlying genetic mechanisms. Several recent reviews cover the biosynthesis of apocarotenoid-derived hormones and signaling molecules and their roles in plant development and growth (Felemban et al., 2019; Fiorilli et al., 2019; Wang et al., 2020b; Moreno et al., 2021). In this review, we will mainly focus on the advances that have recently been made in understanding the regulation, metabolism, and genetic mechanism underlying apocarotenoid diversity, particularly apocarotenoid pigments and volatiles. We also depict the possible impact of understanding the mechanisms behind apocarotenoids diversity on accelerating molecular breeding and CRISPR-based next-generation metabolic engineering of apocarotenoids for crop improvement.

\section{THE SIGNIFICANCE AND FUNCTION OF APOCAROTENOIDS}

Non-enzymatic and enzymatic oxidative cleavage of carotenoids and further metabolism of thereby arising apocarotenoids lead to various biologically important plant metabolites, including phytohormones, pigments, volatiles, and signaling molecules (Table 1). Due to their important roles in many physiological, developmental processes, and plant-biotic environment interactions, the phytohormones ABA and SLs are best-known examples for biologically important plant apocarotenoids. ABA is widely distributed in nature and is common in cyanobacteria, algae, fungi, plants as well as in animals (Wang et al., 2020b). It plays important roles in many physiological and developmental processes, including the establishment of seed dormancy, root, and shoot growth and regulation of stomatal closure, and is a major component in plant abiotic and biotic stress response (Chen et al., 2020; Moreno et al., 2021). SLs were originally discovered as the host-derived rhizospheric chemical signal that induces seed germination in root parasitic plants, such as Striga hermonthica, which represent a major threat to global food security (Xie et al., 2010; Jamil et al., 2021). Later studies on arbuscular mycorrhization revealed the role of SLs as the plantderived signal that induces hyphal branching in AM-fungi, which paves the way for host colonization (Cook et al., 1966; Lanfranco et al., 2018; Wang et al., 2020b). Within plants, SLs inhibit shoot branching and the formation of adventitious roots, stimulate internodes elongation, and increase stem thickness (Al-Babili and Bouwmeester, 2015; Moreno et al., 2021). Furthermore, SLs are involved in leaf and floral organ senescence, as well as in biotic and abiotic stress response (Al-Babili and Bouwmeester, 2015; Decker et al., 2017; Wang et al., 2020b). Besides these two apocarotenoid-derived phytohormones, recent studies on the formation and biological functions of apocarotenoids unraveled new carotenoid-derived plant signaling molecules and growth regulators. For example, Wang et al. (2019a) identified a new growth regulator named zaxinone, which promotes rice growth and modulates its hormone homeostasis. Exogenous application 
TABLE 1 | Biosynthesis and biological functions of plant apocarotenoids and their derivatives.

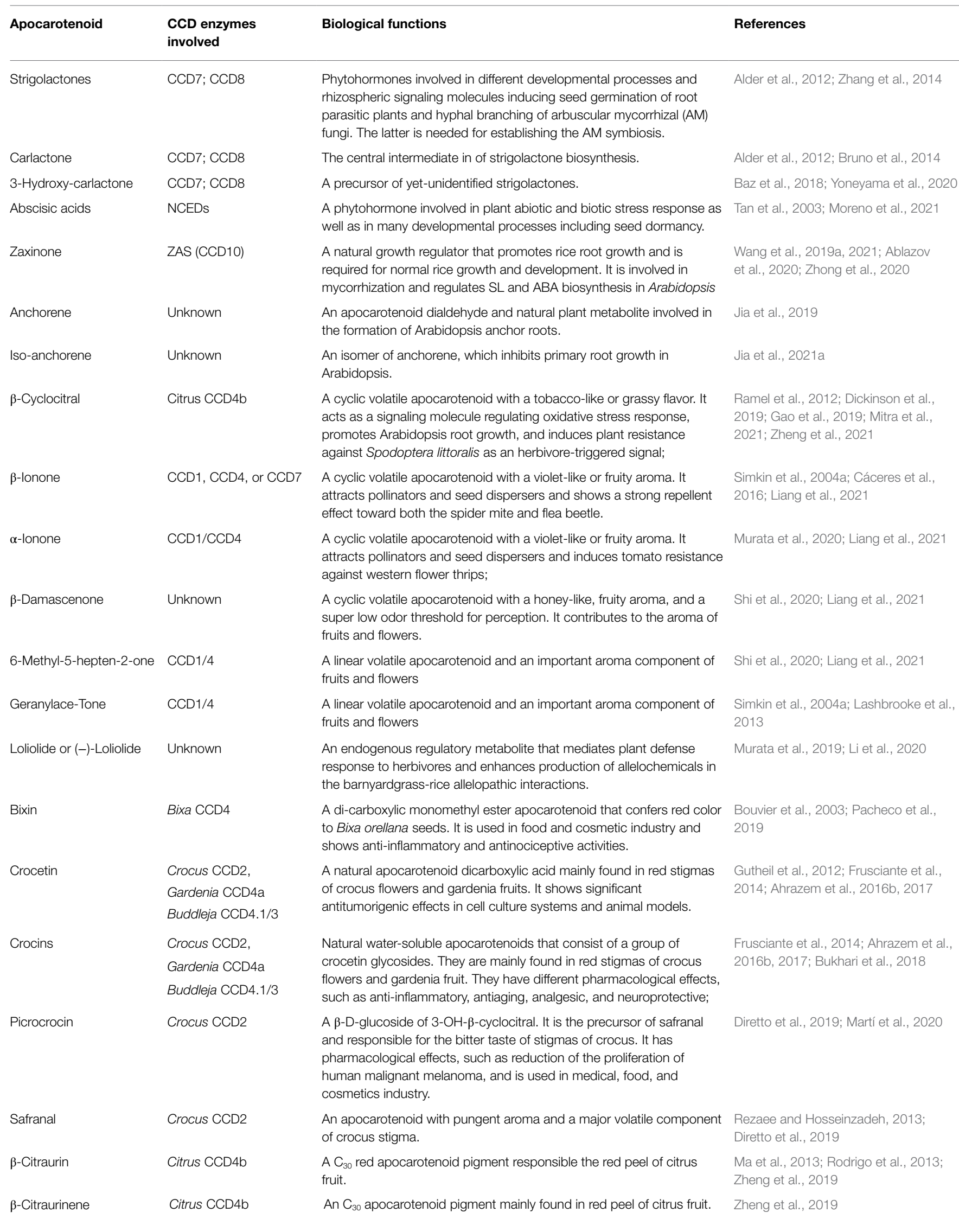


of zaxinone as well as of zaxinone mimics increased the growth of crown roots of rice seedlings and alleviated Striga-infestation in a Striga-susceptible rice cultivar, by reducing SL content and release (Wang et al., 2019a, 2020a), demonstrating its application potential for improving rice growth and combating root parasitic weeds. Very recently, Wang et al. (2021) also demonstrated that increased sugar uptake and metabolism is likely the major reason for growth-promoting activities of zaxinone in rice and that zaxinone effect on root cell division activity in root meristem and on the number of cortex cell layers is likely caused by modulating cytokinin content. The enzyme involved in zaxinone formation in rice, the carotenoid cleavage dioxygenase (CCD) Zaxinone synthase, is common in mycorrhizal plants, but absent in those, such as Arabidopsis thaliana, that do not build this symbiosis (Wang et al., 2019a). In contrast to rice, a recent study showed that zaxinone application to hydroponically grown Arabidopsis seedlings increased SL and ABA content in roots and did not improve growth. This result suggests that the activities and functions of zaxinone may differ between plant species and are likely coupled to their ability to establish arbuscular mycorrhizal fungi (AMF) symbiosis (Wang et al., 2019a; Ablazov et al., 2020; Moreno et al., 2021).

Oxidative cleavage of carotenoids at more than one double bond results in the production of various dialdehyde products. Jia et al. (2021a) identified a carotenoid-derived $\mathrm{C}_{10}$-dialdehyde that was named anchorene, which specifically promoted the growth and development of Arabidopsis anchor roots. This type of roots emerges from the collet region, the root-hypocotyl junction, through modulating auxin homeostasis. The identity of anchorene as a natural carotenoid-derived metabolite was confirmed using mutants affected in carotenoid biosynthesis and chemical inhibitors of carotenoid biosynthesis and by establishing a LC-MS-based system for isolating and detecting of carotenoidderived dialdehydes (Jia et al., 2019; Mi et al., 2019). The discovery of anchorene unraveled, for the first time, a biological function of carotenoid-derived dialdehydes in plant development.

$\beta$-Cyclocitral $(\beta-\mathrm{CC})$ is a volatile apocarotenoid derived from the oxidation of carotenoids (e.g., $\beta$-carotene), which is widely present in nature and is common in various organisms ranging from cyanobacteria and fungi to plants. It was shown that $\beta$-CC is a retrograde signaling molecule regulating the expression of oxidative stress-responsive genes, resulting in acclimation of plants to high-light conditions (Ramel et al., 2012). $\beta$-cyclocitric acid ( $\beta$-CCA), a direct oxidation product of $\beta$-CC, is also a stress signal that enhanced drought tolerance likely through a signaling pathway different from that of $\beta$-CC (D'alessandro et al., 2019). Because of its water-soluble character, $\beta$-CCA is more suitable for agricultural application and can be more easily used to increase drought tolerance of crops through irrigation or by spraying, compared with its precursor $\beta$-CC (Havaux, 2020). In addition to its role in oxidative stress response, $\beta$-CC is a root growth regulator. Exogenous application of $\beta$-CC promoted the growth of primary roots in Arabidopsis, tomato, and rice. It was also shown that $\beta$-CC alleviates the inhibitory effect of salt on the growth of rice roots (Dickinson et al., 2019). A recent study showed that $\beta$-CC induces plant resistance against Spodoptera littoralis, as an herbivore-triggered signal, and downregulates the methylerythritol-4-phosphate (MEP) pathway flux, which is responsible for plastid isoprenoid biosynthesis, through direct inhibition of 1-deoxy- $D$-xylulose-5-phosphate synthase (DXS), a rate-limiting enzyme of the MEP pathway (Mitra et al., 2021). Other volatile apocarotenoids are also involved in herbivore resistance or response. For example, $\beta$-ionone is released as herbivores-induced defense volatile in canola, Brassica napus, and shows a strong repellent effect toward both the spider mite and flea beetle (Cáceres et al., 2016). $\alpha$-Ionone, a structural isomer of $\beta$-ionone, induces jasmonic acid (JA) independent resistance to western flower thrips in tomato and Arabidopsis, by reducing the survival rate of western flower thrips without exhibiting direct insecticidal activity (Murata et al., 2020). Loliolide, a $\mathrm{C}_{11}$-terpene lactone, functions as an endogenous signaling metabolite that induces resistance to multiple herbivores, such as the two-spotted spider mite, common cutworm, and western flower thrips, possibly independent of JA signaling (Murata et al., 2019). Application of loliolide to tomato leaves reduced the survival rate of the two-spotted spider mite and larvae of the common cutworm, without exhibiting direct toxic activity against these herbivores (Murata et al., 2019). Moreover, loliolide [also referred to as (-)-loliolide] was found to act as a soil-borne signaling molecule that enhances the production of allelochemical, such as momilactone B and tricin, to coordinate barnyardgrassrice allelopathic interactions (Kong et al., 2018; Li et al., 2020).

Besides their biological functions within plants, many apocarotenoid volatiles are recognized as important aroma components of different flowers and fruits of horticultural plants (Shi et al., 2020). For instance, linear volatile apocarotenoids, such as geranyl acetone, 6-methyl-5-hepten-2-one (MHO), and geranial, were identified as important aroma components in fruits of papaya, citrus, and blackberry (González-Mas et al., 2011; Jing et al., 2015; Reidel et al., 2016). Cyclic apocarotenoids, such as $\beta$-CC, $\beta$-ionone, and $\beta$-damascenone, have lower odor thresholds and stronger impacts on people's aroma perception, compared with linear volatiles (Goff and Klee, 2006). Therefore, cyclic apocarotenoids are recognized as more important volatiles with respect to the aromatic odor of fruits and flowers (Shi et al., 2020). The $C_{13}$ cyclic $\beta$-damascenone has an attractive honey-like, floral, and fruity flavor and shows a very low odor threshold of $2 \mathrm{ng} / \mathrm{L}$ on people's aroma perception, hence, it has been frequently used as flavoring ingredient in fragrance industry (Liang et al., 2021). The cyclic volatiles $\alpha$ - and $\beta$-ionone have violet-like, woody, and fruity odor, while $\beta$-CC has a grassy, woody, tobacco-like odor. Both of them are widely used as flavor molecules in fragrances. In addition, these apocarotenoids volatiles also function as effective attractants for pollinators and seed dispersers (Liang et al., 2021).

Apart from being aroma compounds, apocarotenoids can contribute to the color of flowers and fruits and act as antioxidants. Indeed, some long-chain apocarotenoids and their derivatives are used as high-value food colorants, cosmetic agents, and antioxidants and have an important impact in promoting human health. $\beta$-citraurin (3-hydroxy- $\beta$-apo- $8^{\prime}$ carotenal) and $\beta$-citraurinene are two major $\mathrm{C}_{30}$ apocarotenoid pigments found in Citrus, and their hyper-accumulation is responsible for the attractive red coloration of fruit peel in 
some citrus species (Zheng et al., 2019). Bixin $\left(C_{25}\right)$ is a further natural apocarotenoid pigment that confers red color to $B$. orellana seeds. It has been widely extracted from natural sources and used in food and cosmetic industry as a color additive (Giuliano et al., 2003b). Furthermore, Pacheco et al. (2019) demonstrated anti-inflammatory and antinociceptive activities of bixin, which were shown to be caused by reduction of neutrophil migration in simulated wound tests in rats. The proposed biosynthetic pathway of bixin is supposed to start with the cleavage of all-trans-lycopene into bixin aldehyde, followed by three sequential conversion steps catalyzed by the bixin aldehyde dehydrogenase and nor-bixin carboxyl methyltransferase (Bouvier et al., 2003). Saffron is one of the oldest natural food additives and worldwide the most expensive spice. It corresponds to the stigma of saffron flowers, which accumulates the $3-\mathrm{OH}-\beta$-cyclocitral glycoside picrocrocin, the $\mathrm{C}_{10}$ volatile apocarotenoid safranal, and the $\mathrm{C}_{20}$ pigment crocetin and its glycosides crocins. Crocetins and crocins are responsible for the red color, while picrocrocin and safranal give rise for the bitter taste and pungent aroma of saffron (Frusciante et al., 2014). These apocarotenoids are beneficial to health and are commonly used in food and cosmetics in many countries. Saffron apocarotenoids were also shown to have several pharmacological effects, such as anti-inflammatory, antiaging, analgesic, and neuroprotective (Bukhari et al., 2018).

\section{THE METABOLIC BACKGROUND OF PLANT APOCAROTENOIDS STRUCTURAL DIVERSITY}

\section{Various Carotenoid Cleavage Dioxygenases Mediate Site-Specific Oxidative Tailoring of Carotenoids, Forming a Diverse Set of Apocarotenoids}

In plants, the enzymatic cleavage of carotenoids at specific positions is generally catalyzed by CCDs, non-heme irondependent enzymes present in plants, animals, bacteria, and fungi (Giuliano et al., 2003a; Sui et al., 2013; Ahrazem et al., 2016a). In the last decades, different types of plant CCDs with distinct features have been identified and investigated. Arabidopsis contains five types of CCD enzymes, known as CCD1, CCD4, CCD7, CCD8, and 9-cis-epoxycarotenoid dioxygenase (NCED). There are five NCEDs in Arabidopsis, NCED2, NCED3, NCED5, NCED6, and NCED9, while the other types are represented by a single enzyme each (Auldridge et al., 2006; Walter and Strack, 2011). The five NCEDs catalyze the oxidative cleavage of 9-cis-violaxanthin and/or 9'-cis-neoxanthin to produce xanthoxin, the precursor of ABA (Figure 1; Schwartz et al., 1997; Tan et al., 2003; Al-Babili and Bouwmeester, 2015; Jia et al., 2018). This apocarotenoid product is exported from plastids, the site of carotenoid biosynthesis and most of plant cleavage enzymes, to the cytosol and converted to abscisic aldehyde by the enzyme short-chain dehydrogenase (SDR; González-Guzmán et al., 2002). The arising abscisic aldehyde is then oxidized by a molybdenum-dependent aldehyde oxidase
(AAO) to produce ABA (Seo et al., 2000). Very recently, Jia et al. (2021b) found that $\beta$-apo-11-carotenoids $\left(C_{15}\right)$, i.e., $\beta$-apo11-carotenal, 9-cis- $\beta$-apo-11-carotenal, 3-OH- $\beta$-apo-11-carotenal, and 9-cis-3-OH- $\beta$-apo-11-carotenal, exert ABA-like biological functions in maintaining seed dormancy and inducing the expression of ABA-responsive genes. Further feeding experiments with labeled apocarotenoids, combined with analysis of physiological and transcriptional responses, showed that plants can synthesize $\mathrm{ABA}$ from $\mathrm{C}_{15} \beta$-apo-11-carotenoids, which represents an alternative, zeaxanthin epoxidase-independent ABA biosynthetic pathway (Jia et al., 2021b). The genes involved in the hydroxylation, isomerization, and oxidation the $\mathrm{C}_{15}$ $\beta$-apo-11-carotenoids in this new ABA biosynthesis pathway remain elusive, which would be important for future exploration of the biological significance of this route.

The other four CCDs of Arabidopsis, CCD1, CCD4, CCD7, and CCD8 show different cleavage activities and biological functions (Zheng et al., 2020a). CCD7 and CCD8 are involved in SL biosynthesis. CCD7 cleaves 9 -cis- $\beta$-carotene formed by the cis-trans-isomerase D27 (Alder et al., 2012; Bruno et al., 2014; Bruno and Al-Babili, 2016; Abuauf et al., 2018) to produce the apocarotenoids 9 -cis- $\beta$-apo- $10^{\prime}$-carotenal $\left(\mathrm{C}_{27}\right)$ and $\beta$-ionone $\left(\mathrm{C}_{13}\right)$. CCD8 catalyzes a combination of reactions converting 9-cis- $\beta$-apo- $10^{\prime}$-carotenal into carlactone, the central intermediate in SL biosynthesis (Figure 1; Alder et al., 2012; Bruno et al., 2014; Bruno and Al-Babili, 2016; Abuauf et al., 2018). Thereby, it is assumed that CCD8 mediates isomerization, intramolecular rearrangement, and repeated oxygenation reactions (Alder et al., 2012; Bruno et al., 2017).

Plant CCD1 enzymes, characterized by their relaxed cleavage site and substrate specificity, cleave linear, monocyclic, and bicyclic carotenoid and apocarotenoid substrates at different double bonds (Figure 2), yielding various apocarotenoid aldehydes and ketones with different chain lengths (Schwartz et al., 2001; Vogel et al., 2008; Ilg et al., 2009, 2014). Some CCD1 cleavage products are volatile compounds that contribute to the aroma and flavor of fruits and flowers of important horticulture crops. For example, CCD1-catalyzed C9-C10 and/or C9'-C10'cleavage of carotenoids forms $\mathrm{C}_{14}$ dialdehyde and $\mathrm{C}_{13}$ ketone products (Figure 2), including $\beta$-ionone, $\beta$-ionophore, $\alpha$-ionone, pseudoionone, and geranylacetone, which are flavor and fragrance volatiles in fruits or flowers of many different plant species, such as tomato (Simkin et al., 2004a), grape (Mathieu et al., 2005), melon (Ibdah et al., 2006), and petunia (Simkin et al., 2004b). CCD1 was also shown to cleave lycopene at the C5-C6 and/or $\mathrm{C}^{\prime}$ - $\mathrm{C6}^{\prime}$ position to yield $\mathrm{MHO}$ (Figure 2), an important aroma compound in tomato (Vogel et al., 2008). Additionally, the rice CCD1 enzyme showed an in vitro enzymatic activity that targets the C7-C8 bond of lycopene (Figure 2), generating a $\mathrm{C}_{10}$ flavor compound, geranial (Ilg et al., 2009). However, CCD1 enzymes are localized to cytoplasm, therefore they seem to cleave already destructed carotenoids transported to cytoplasm (i.e., apocarotenoids) rather than carotenoid substrates in plastid (Ilg et al., 2009, 2010). Consistent with this speculation, overexpression of rice CCD1 in Golden Rice did not lead to significant decrease of the carotenoid content in endosperm (Ilg et al., 2010). 
CCD2, a specific CCD type that is restricted to the Crocus and Freesia genus of the Iridaceae family, is closely related to the cytoplasmic CCD1 subfamily, although it is localized in plastids (Frusciante et al., 2014; Ahrazem et al., 2016b; Fang et al., 2020). Crocus CCD2 was shown to cleave the C7-C8 and $\mathrm{C7}^{\prime}-\mathrm{C}^{\prime}$ double bonds of zeaxanthin to form crocetin dialdehyde $\left(\mathrm{C}_{20}\right)$ and 3-OH- $\beta$-cyclocitral $\left(\mathrm{C}_{10}\right.$; Frusciante et al., 2014; Ahrazem et al., 2016b), which are subsequently converted to crocins and safranal (Figure 3), respectively, contributing to the color and aroma of saffron stigma (Demurtas et al., 2018; Diretto et al., 2019).

Carotenoid cleavage dioxygenase $4 \mathrm{~s}$ are a further type of plastid-localized CCDs. They are involved in the production of apocarotenoid volatiles and their activity determines carotenoid content and apocarotenoid pigments accumulation (Ohmiya et al., 2006; Campbell et al., 2010; Brandi et al., 2011; GonzalezJorge et al., 2013; Zhang et al., 2015). CCD4 enzymes from different plant species vary in their substrate specificity and regioselectivity (Figure 3; $\mathrm{Mi}$ and Al-Babili, 2019). In Arabidopsis and potato, CCD4 was shown to catalyze the cleavage of bicyclic carotenoids at the $\mathrm{C} 9-\mathrm{C} 10$ or $\mathrm{C}^{\prime}-\mathrm{C} 10^{\prime}$ double bond to produce $\mathrm{C}_{13}$ volatiles, and $\mathrm{C}_{27}$ apocarotenoids that are supposed to be further degraded to smaller metabolites (Figure 3; Bruno et al., 2016). This enzymatic activity of CCD4 decreases carotenoid content in various tissues of plant species, including potato tubers, Chrysanthemum and Brassica flowers, Japanese morning glory petals, peach fruits, and Arabidopsis seeds (Ohmiya et al., 2006; Campbell et al., 2010; Brandi et al., 2011; Falchi et al., 2013; Gonzalez-Jorge et al., 2013; Zhang et al., 2015; Watanabe et al., 2018; Han et al., 2019a). In contrast, the Citrus CCD4b targets at a different site, i.e., the $\mathrm{C} 7-\mathrm{C} 8$ or $\mathrm{C}^{\prime}-\mathrm{C}^{\prime}$ double bond, of $\beta$-carotene, $\beta$-cryptoxanthin, and zeaxanthin to form $\mathrm{C}_{30}$ apocarotenoid pigments, such as $\beta$-citraurin, together with $\mathrm{C}_{10}$ volatiles, such as $\beta$-CC in citrus peel (Figure 3; Ma et al., 2013; Rodrigo et al., 2013; Zheng et al., 2015, 2019, 2021). It is believed that Citrus CCD4b is also involved in the production of $\beta$-citraurinene and $\beta$-citraurol, two further Citrus fruit-specific $\mathrm{C}_{30}$ apocarotenoid pigments in citrus peels (Zheng et al., 2021). Indeed, $\mathrm{C}_{30}$ apocarotenoids are the main long-chain apocarotenoids in $C C D 4 b$-overexpressing citrus callus, reinforcing the major roles of $\mathrm{CCD} 4 \mathrm{~b}$ in the biosynthesis of $\mathrm{C}_{30}$ pigments in citrus peel (Zheng et al., 2019, 2021). The BdCCD4.1 and BdCCD4.3 from Buddleja davidii, showing the same enzymatic activity of crocus $\mathrm{CCD} 2$, cleave $\mathrm{C} 7-\mathrm{C} 8$, and $\mathrm{C}^{\prime}-\mathrm{C}^{\prime}$ double bonds of zeaxanthin in vitro and in vivo, to form crocetin dialdehyde and 3-hydroxy- $\beta$-cyclocitral (Ahrazem et al., 2017). A recent study reported another CCD4 type from Gardenia jasminoides

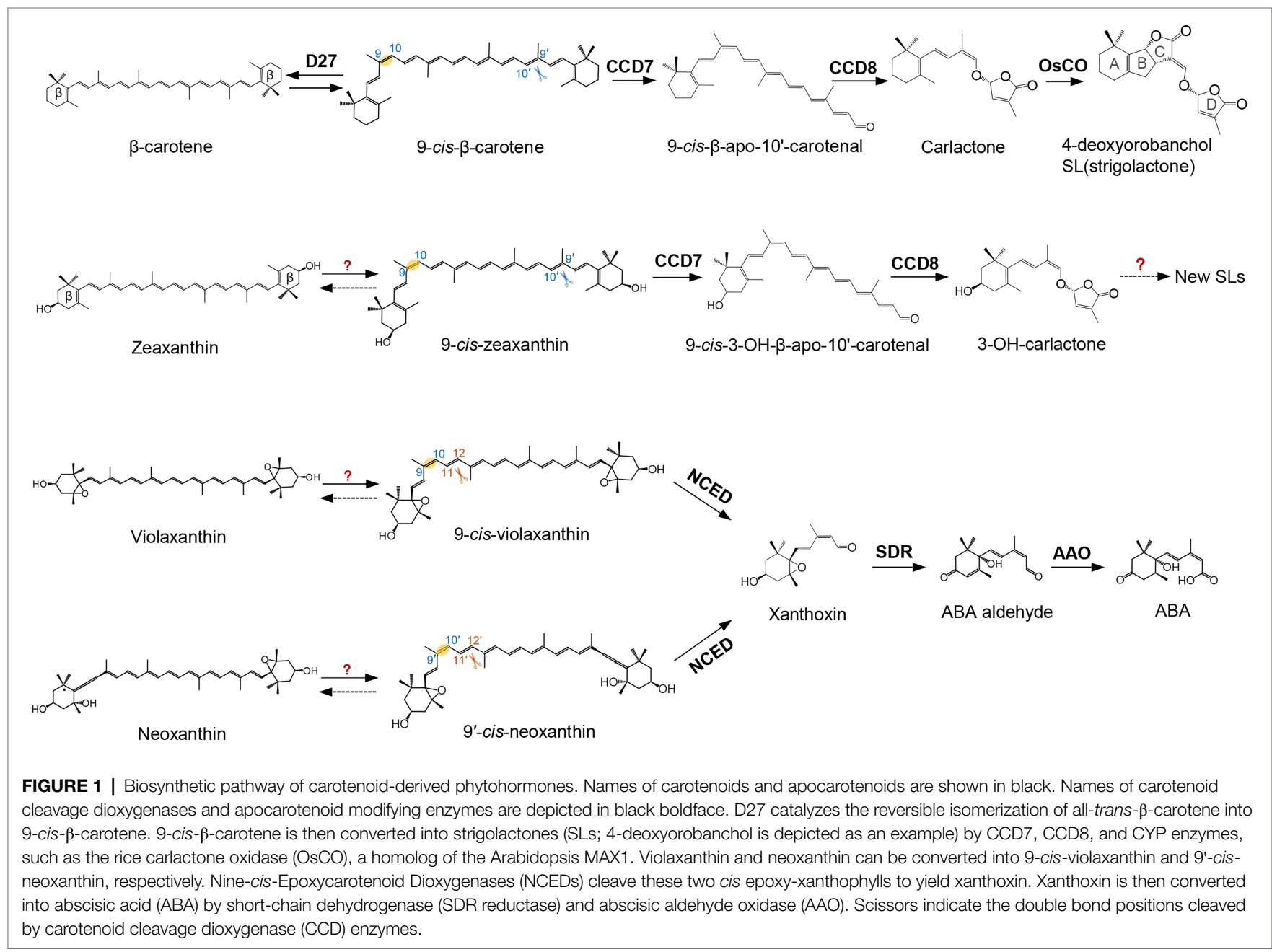


that cleaves several substrates, including $\beta$-carotene, zeaxanthin, and lycopene, at both $\mathrm{C} 7-\mathrm{C} 8$ and $\mathrm{C}^{\prime}-\mathrm{C} 8^{\prime}$ double bonds, and is responsible for the production of crocetin and crocins in gardenia fruit (Figure 3; Xu et al., 2020b).

Zaxinone synthase (ZAS), also referred to as CCD10, is a new CCD-type that is missing in Arabidopsis and which has been recently unraveled in rice and maize (Wang et al., 2019a; Zhong et al., 2020). The rice ZAS was shown to cleave the 3-OH-apocarotenoids with different chain lengths, such as $\mathrm{C}_{30}$ all-trans-3-OH-apo-8'-carotenal, $\mathrm{C}_{27}$ all-trans-3-OH-apo-10'carotenal, and $\mathrm{C}_{25}$ all-trans-3-OH-apo-12'-carotenal, at the C13-C14 double bond to yield a $\mathrm{C}_{18}$-ketone product, zaxinone, that is characterized as a signaling molecule regulating plant growth and development (Wang et al., 2019a). It seems likely that the presence of this enzyme correlates with the ability to establish mycorrhizal symbiosis, as non-mycorrhizal plants, such as Brassica species, do not contain this type of CCDs.

\section{Unspecific Oxidative Cleavage Processes in the Formation of Apocarotenoids}

There are other enzymes mediating carotenoid cleavage, besides CCDs. For instance, lipoxygenases (LOXs) use carotenoids as co-substrates, however, resulting in unspecific oxidative cleavage of carotenoids. The fatty acid peroxyl radicals produced by LOXs enzymatic peroxidation of polyunsaturated fatty acids, such as linolenic, arachidonic, and linoleic acid, can attack carotenoids, leading to their degradation, as was shown in vitro (Jarén-Galán and Mínguez-Mosquera, 1999). Disruption and downregulation of $L O X$ genes reduced carotenoid degradation during pasta processing and decreased the degradation of carotenoids in Golden Rice during storage, respectively (Carrera et al., 2007; Gayen et al., 2015), reinforcing the role of LOXs in carotenoid oxidative cleavage. Recently, by using pan-genome analysis, quantitative trait locus mapping and functional analysis, Gao et al. found that one tomato LOX (referred to as TomLoxC) is responsible for the production of apocarotenoid volatiles, including $\beta$-CC and $\beta$-ionone, in tomato fruits (Gao et al., 2019).

In addition to unspecific enzymatic degradation processes, carotenoids are subjected to unspecific non-enzymatic destructive oxidation triggered by reactive oxygen species (ROS; Ramel et al., 2012; Hou et al., 2016), which initiates metabolic pathways yielding various signaling molecules and/or volatile compounds. For example, singlet oxygen formed in chloroplasts, particularly at photosystem (PS) II under high-light condition, attacks all-trans $\beta$-carotene, giving rise to the formation of various
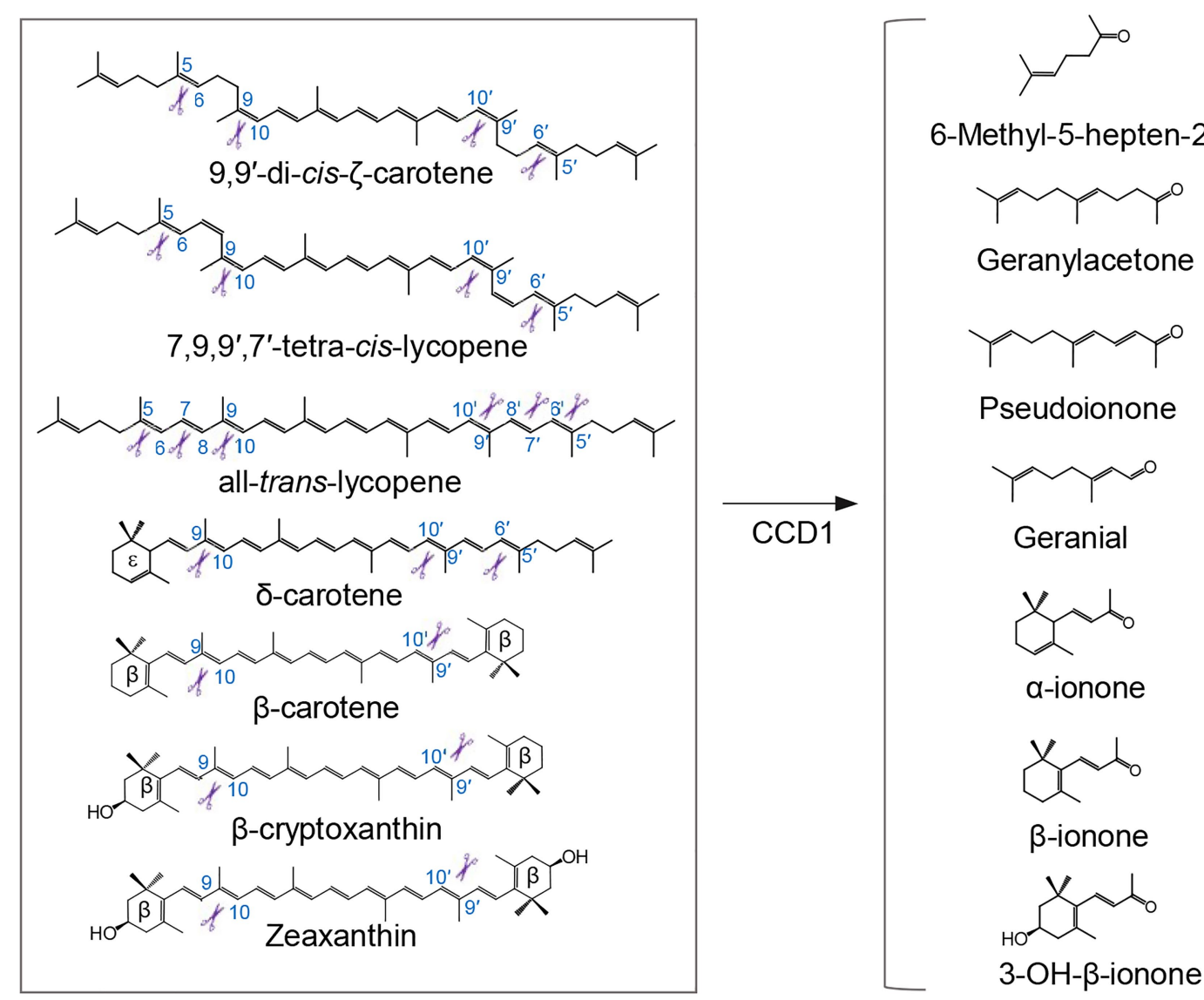

FIGURE 2 | Cleavage of carotenoid substrates by CCD1 enzyme and examples of generated volatiles. Plant CCD1 enzymes can cleave linear, monocyclic, and bicyclic carotenoid and apocarotenoid substrates at different double bonds in E. coli and/or in vitro, yielding various apocarotenoid volatiles, in addition to a plentitude of apocarotenoid dialdehydes (not shown). Scissors indicate the double bond positions cleaved by CCD1. 


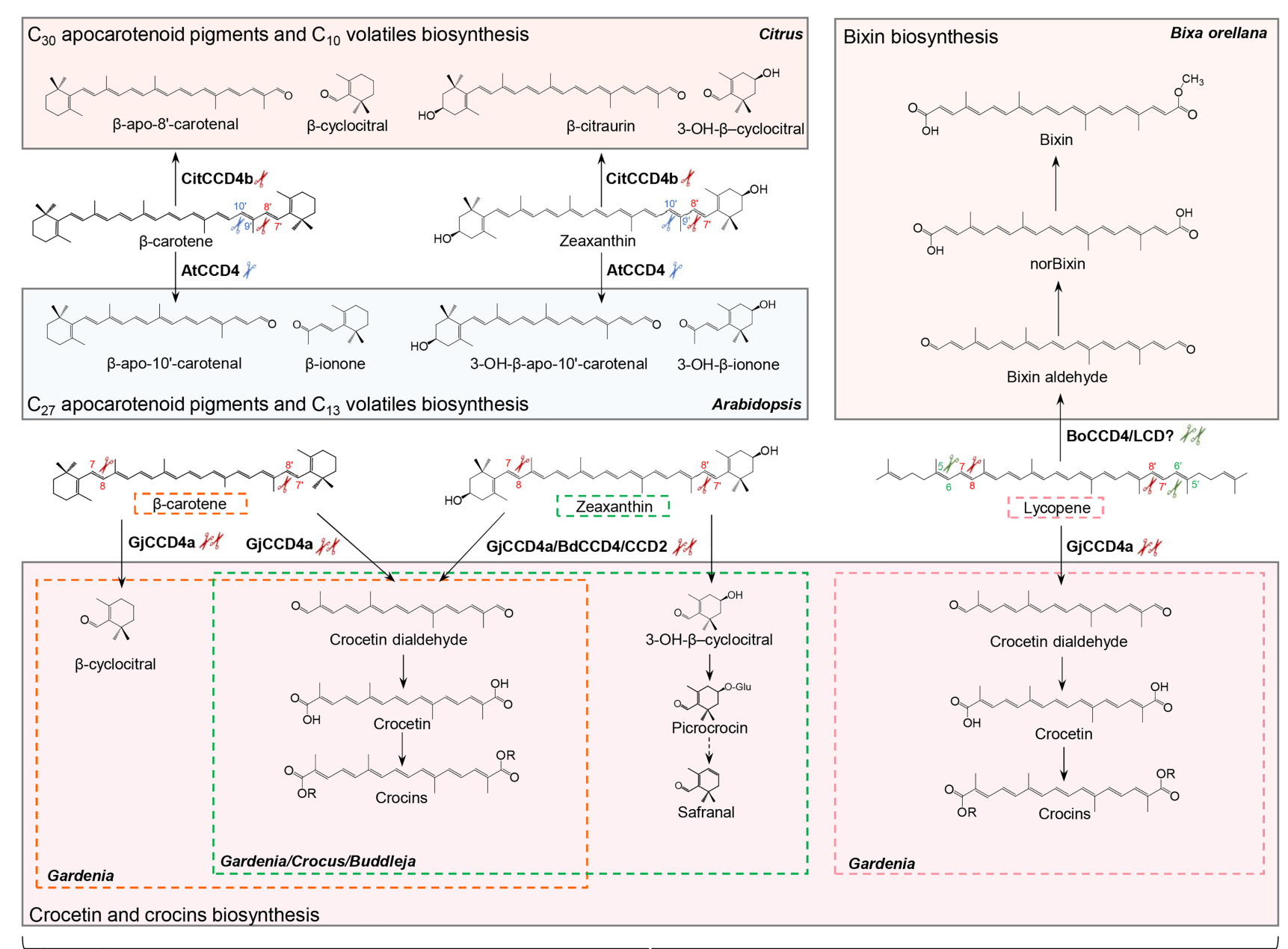

Diversity of plant CCD4s in substrate and cleavage-site specificity

FIGURE 3 | Diversity of carotenoid cleavage dioxygenase 4 (CCD4) cleavage reactions. Names of apocarotenoids are shown in black. Names of carotenoid cleavage dioxygenases are in depicted black boldface. Scissors in different color indicate the positions cleaved by CCD4 or CCD2 enzymes from different plant species. Abbreviations: CitCCD4b, Citrus CCD4b; AtCCD4, Arabidopsis thaliana CCD4; GjCCD4a, Gardenia jasminoides CCD4a; BdCCD4, Buddleja davidii CCD4, CsCCD2, Crocus sativus CCD2; and BoCCD4/LCD, Bixa orellana CCD4/LCD.

apocarotenoid aldehydes, ketones, endoperoxides, epoxides, and lactones (Stratton et al., 1993; Hasegawa et al., 2004; Fiedor et al., 2005; Yamauchi et al., 2014), including the growth regulator and signaling molecule, $\beta$-CC (Ramel et al., 2012; Dickinson et al., 2019). It is assumed that linear volatile apocarotenoids, such as MHO, citral, geranylacetone, pseudoionone, and farnesylacetone, can also be generated through the unspecific oxidative degradation of carotenoid precursors, besides the possible involvement of CCD1 enzymes (Simkin et al., 2004a; Moreno et al., 2021).

\section{MOLECULAR REGULATION OF APOCAROTENOID BIOSYNTHESIS}

The production of plant apocarotenoids varies depending on developmental stage and tissue and is affected by environmental stimuli that lead to non-enzymatic cleavage of carotenoid. Due to their role as regulatory metabolites, the genesis of several apocarotenoids is tightly regulated at different levels, including transcriptional, post-translational, and epigenetic regulation.

\section{Transcriptional Regulation}

Many studies have reported that some CCD genes show tissue/ organ-specific expression pattern that also depends on growth/ developmental stages and environmental conditions (GarcíaLimones et al., 2008; Chiou et al., 2010; Gonzalez-Jorge et al., 2013; Yuan et al., 2015; Zheng et al., 2019). Several transcriptional regulators are known to regulate the expression of genes related to apocarotenoid production in flowers and fruits of horticulture crops. For instance, sweet osmanthus (Osmanthus fragrans) OfWRKY3 and OfERF61 transcription factors were shown to directly bind to the CAACA and W-box elements in OfCCD4 promoter, which results in an increase of OfCCD4 expression 
level (Han et al., 2016, 2019b). It is assumed that these two transcription factors are positive regulators of the biosynthesis of $\beta$-ionone, a key aromatic component in sweet osmanthus petals, which is formed through OfCCD4 cleavage reaction (Huang et al., 2009; Han et al., 2016, 2019b). In grapes, the expression level of $V v C C D 4 b$ was shown to positively correlate with the content of several apocarotenoid volatiles, including $\mathrm{MHO}, \beta$-ionone, and $\beta$-damascenone. $V v M A D S 4$, a MADS family transcription factor, directly binds to the promoter of $V v C C D 4 b$ and represses its expression (Meng et al., 2020). Transcription factors that activate apocarotenoid biosynthetic genes, in some cases, also increase the expression of carotenoid biosynthetic genes responsible for the production of the carotenoid substrates. For instance, the citrus CsMADS6 was shown to directly bind to and to activate the promoters of citrus CCD1, as well as those of carotenoid biosynthetic genes, such as LCYb1 (Lycopene $\beta$-cyclase 1), PSY (phytoene synthase), and PDS (phytoene desaturase; Lu et al., 2018). Very recently, Zhu et al. (2021) found that citrus CsERF061 enhances the expression levels of CCD1, NCED3, and $C C D 4$ by direct binding to their promoters and to those of the seven carotenoid biosynthetic genes, PSY1, PDS, carotene isomerase (CRTISO), LCYB1, LCYB2, $\beta$-carotene hydroxylase $(B C H)$, and zeaxanthin epoxidase (ZEP), indicating the involvement of CsERF061 in a multi-target regulation of carotenoid and apocarotenoid metabolism.

The CsULT1 Ultrapetala transcription factor, identified in crocus, was found to positively regulate the gene expression of CCD2 and CCD4b, as well as of PSY, PDS, and $B C H$ genes (Ashraf et al., 2015).

Unspecific enzymatic cleavage of carotenoids is also regulated at transcriptional levels. In tomato, overexpression of SlMYB75, a MYB-type transcription factor, resulted in significant increase in the expression level of LOXC gene that is involved in unspecific enzymatic production of volatile apocarotenoids (e.g., $\beta$-CC). Yeast one-hybrid assay revealed that SIMYB75 can directly bind to MYBPZM and MYBPLANT cis-elements in $L O X C$ promoter. Further dual-luciferase assays proved the role of SIMYB75 in activating the promoters of the LOXC (Jian et al., 2019).

It should be mentioned that although these transcription factors have been shown to regulate the expression levels of carotenoid and/or apocarotenoid biosynthetic genes, their effects on the apocarotenoid content and composition in target tissues are still poorly understood. Further quantification of apocarotenoid pigments and/or volatiles by LC-MS/GC-MS profiling should be performed in plants overexpressing or lacking these transcription factors, to confirm their contribution to apocarotenoid production.

As it can be expected, apocarotenoid-derived hormones biosynthesis is also regulated by transcriptional factors in various tissues/organs. For example, basic pentacysteine 1 (BPC1) can bind to the promoter of CCD7, and transient overexpression of $B P C 1$ repressed CCD7's promoter activity in roots of Malus baccata (Yue et al., 2015). The CDF4 transcription factor from Arabidopsis, a DOF family protein, is a positive regulator of $\mathrm{ABA}$ biosynthesis and leaf senescence, which increases the expression of NCED2 and NCED3 by directly binding to their promotors (Xu et al., 2020a). A NAC transcription factor, ATAF1, from Arabidopsis was shown to bind to the promoter of NCED3 in vivo and to activate its expression, which resulted in increased ABA levels. Moreover, a rice $N A C$ transcription factor OsNAC2 also upregulates the expression of OsNCED3 as well as OsZEP1 and represses the expression of the ABA catabolic gene OsABA8ox1, which results in an increase of ABA levels and accelerated leaf senescence. In peach fruit, the ethylene response transcription factor PpERF3 was found to bind to the promoters of PpNCED2/3 genes and to promote their expression, which results in an increased ABA content during fruit ripening (Wang et al., 2019b). In Citrus reticulata fruit, the $\mathrm{R} 2 \mathrm{R} 3-\mathrm{MYB}$ transcriptional factor CrMYB68 is a negative regulator of $\mathrm{CrBCH} 2$ and $\mathrm{CrNCED} 5$ gene expression, contributing to the delay in the biosynthesis of ABA in "Green Ougan," a stay-green mutant of C. reticulata cv. Suavissima (Zhu et al., 2017). CrMYB68 also interacts with a novel NAC transcription factor, CrNAC036, during binding to the promoter of CrNCED5, causing a synergetic effect in inhibiting ABA biosynthesis in $C$. reticulata fruits (Zhu et al., 2020). Very recently, a citrus homeodomain leucine zipper I transcription factor called CsHB5 was found to positively regulate $\mathrm{ABA}$ accumulation and senescence, by directly binding to the promoters of carotenoid and $\mathrm{ABA}$ biosynthetic genes, including $\mathrm{BCH}$ and $\mathrm{NCED} 2$, and activating their transcription (Zhang et al., 2021). In Arabidopsis seeds, the basic helixloop-helix transcription factor bHLH57 was shown to induce the expression of NCED6 and NCED9 through binding to the E-box (CANNTG)/G-box (CACGTG) motifs within their promoters, thereby leading to higher $A B A$ levels that impose seed dormancy. In contrast, reversal of rdo5 (ODR1), a Homolog of Rice Seed Dormancy4, was shown to decrease seed dormancy by directly interacting with bHLH57 and inhibiting the bHLH57mediated induction of NCED6 and NCED9 in the nucleus (Liu et al., 2020).

\section{Post-translational Regulation}

Post-translational regulation via protein-protein interactions provides an alternative mechanism to regulate CCD enzymatic activity in plants, thereby regulating the content and composition of apocarotenoids. PGM48, a plastoglobule (PG)-localized metallopeptidase identified in Arabidopsis, was found to positively regulate leaf senescence (Bhuiyan et al., 2016; Bhuiyan and van Wijk, 2017). PGM48 is the only protease present in PG proteomes. The protein level of PGM48 increased significantly during leaf senescence, whereas PG-localized CCD4 decreased. Indeed, overexpression of PGM48 in Arabidopsis dramatically reduced protein levels of CCD4. Moreover, it was shown that PGM48 can directly interact with CCD4, suggesting that CCD4 could be a substrate of PGM48 (Bhuiyan et al., 2016; Bhuiyan and van Wijk, 2017). The direct interaction and co-localization of PGM48 and CCD4 provided new insights into posttranslational regulation of CCD enzymes; however, the role of PGM48 on the production of apocarotenoids is still elusive. Further apocarotenoids profiling and comprehensive characterization of the peptidase activity of PGM48 are still needed (Bhuiyan et al., 2016; Bhuiyan and van Wijk, 2017). 
Plastid-localized ORANGE (OR), a DnaJ cysteine-rich protein, was found to act as a post-translational regulator of the protein level and enzymatic activity of PSY and a determinant of carotenoid accumulation (Zhou et al., 2015; Park et al., 2016; Chayut et al., 2017). Recent evidence showed that OR protein of sweet potato (IbOR) can interact not only with the known target PSY, but also with a new target, CCD4, suggesting that IbOR might also affect carotenoid cleavage as an important factor in carotenoid homeostasis (Park et al., 2020). However, the impact of this interaction on CCD4 protein level or activity is still elusive.

\section{Epigenetic Modification}

Epigenetic regulation is a further way deployed by plants to regulate apocarotenoid biosynthesis. For example, methylation analysis of Oncidium OgCCD1 promoters of white-colored "Jade" and yellow-colored "Gower Ramsey" (GR) cultivars showed that a high level of DNA methylation in GR OgCCD1 promoter, which is consistent with a silent $O g C C D 1$ and higher content of carotenoids in yellow floral tissues of GR (Chiou et al., 2010). In citrus callus, treatment with the DNA methyltransferase inhibitor 5-azacytidine resulted in a dramatic decrease of carotenoid content along with significant upregulation of CPCCD1 gene (Xu et al., 2017). However, in this study, the tested promoter regions of CPCCD1 were not demethylated by the 5-azacytidine effect, suggesting that methylation changes may indirectly regulate expression of CPCCD1 (Xu et al., 2017). Chiou et al. (2010), found that the Oncidium OgCCD1 promoter region of yellow-colored GR cultivar showed a higher level of DNA methylation than that in "Jade," which may result in the downregulation of $O g C C D 1$ in GR, thus leading to less conversion of carotenoids to colorless apocarotenoid in floral tissue. Moreover, a METHYLTRANSFERASE1 (SIMET1) acts as facilitator for SINCED gene expression via directly altering methylation levels of SINCED promoter region, thereby affecting ABA production in tomato epimutant Colorless non-ripening fruits (Yao et al., 2020).

\section{GENETIC INSIGHTS INTO NATURAL VARIATION OF APOCAROTENOID}

Many crop plants, especially horticulture crops, show large variation in quantity and types of apocarotenoids among different species and/or different natural accessions of a single species. For instance, different papaya accessions display big natural variation in accumulating MHO and $\beta$-ionone in fruits (Jing et al., 2015). Farnesyl acetone, $\alpha$-ionone, and $\beta$-ionone show also high natural variation among orange-colored "Nairobi" and "Rothild," yellowcolored "Yellowstone," purple "Purple Haze," and white "Creme de Lite" carrot accessions (Yahyaa et al., 2013). As direct non-enzymatic degradation is supposed to be a major source of apocarotenoids, different concentration of carotenoids may be the reason for the diversity in apocarotenoid levels; however, more and more studies reported that some genetic factors can also determine the apocarotenoid variation, independent of carotenoid levels. For example, in citrus, $\alpha$-ionone, geranial, and $\beta$-ionone are common in "Temple" hybrid mandarin fruit, but cannot be detected in "Murcott" hybrid that even contains significantly higher carotenoid amounts (Yu et al., 2015), In apricot fruit, $\beta$-ionone and dihydro- $\beta$-ionone are considered as two of the major aroma compounds for fruit quality by odor activity values (OVA) method. Contents of $\beta$-ionone and dihydro- $\beta$-ionone were found to be significantly higher in pulps of white apricot cultivars, such as "Luntaixiaobaixing" and "Baixing," than in those of the yellow "Hongyuxing" and "Danxing" cultivars that even accumulate higher carotenoid content (Xi et al., 2016). This finding suggests that the variation of apocarotenoid content in different apricot species is also genetically controlled, other than only determined by the concentration of the carotenoid precursors.

Technological advancements in high-throughput sequencing, metabolomics, and data mining have enabled the integration of various omics datasets, such as genomics, transcriptomics, and metabolomics (Figure 4). The multi-omics strategy coupled with genetic and functional analysis has facilitated exploiting the natural variation of apocarotenoid pigments and volatiles, which paves the way new discoveries in metabolism, function, and evolution evolutionary of plant apocarotenoids.

For instance, Gao et al. (2019) constructed a tomato pan-genome by using genome sequence datasets of 725 tomato accessions. Comparative analyses using this constructed tomato pan-genome identified a rare allele in cultivated tomatoes defined by promoter variation, i.e., $\mathrm{a} \sim 4-\mathrm{kb}$ substitution in the promoter region, of TomLoxC gene encoding a 13-lipoxygenase. Further RNA-Seq data analysis showed that accessions containing both this rare allele captured by pan-genome and another allele present in the "Heinz 1706" genome exhibit significantly higher expression levels of TomLoxC gene than that in other accessions. Further metabolites, QTL and gene expression analysis provided strong evidence that TomLoxC variation is a genetic control for natural variation in the accumulation of nine apocarotenoid volatiles common in tomato fruits and might additionally play an important role in apocarotenoid volatiles biosynthesis. The levels of specific apocarotenoid volatiles were also significantly decreased in transgenic tomato fruits with reduced expression of TomLoxC and in two knock-out Arabidopsis mutants of AtLOX2, the closest TomLoxC homolog. These experiments further confirm that $L O X$ is a genetic determinant for natural variation of apocarotenoid formation and also reinforce the significance of alternative apocarotenoid biosynthesis route via unspecific oxidation process, besides CCD-mediated cleavage reaction. Apocarotenoid volatiles are important quality trait for tomato fruit and flavor (Tieman et al., 2017; Gao et al., 2019). However, this TomLoxC promoter allele captured by pan-genome displays strong negative selection during domestications. This may be due to the primary focus of modern breeding on tomato yield and on resistance to stresses and long shelf-life, which did not consider aroma quality traits.

In addition to volatiles, several recent studies performed apocarotenoid pigments profiling in fruits and related natural variation in some important horticulture crops, such as Citrus and Capsicum species. Citrus fruits show peel color variation among different cultivars. Besides carotenoid content and composition, the production of $\mathrm{C}_{30}$ apocarotenoid pigments, such as $\beta$-citraurin, is also critical for peel pigmentation of citrus mature fruit (Ma et al., 2013; Rodrigo et al., 2013; Zheng et al., $2015,2019,2021)$. Indeed, the content of $\mathrm{C}_{30}$ apocarotenoid 


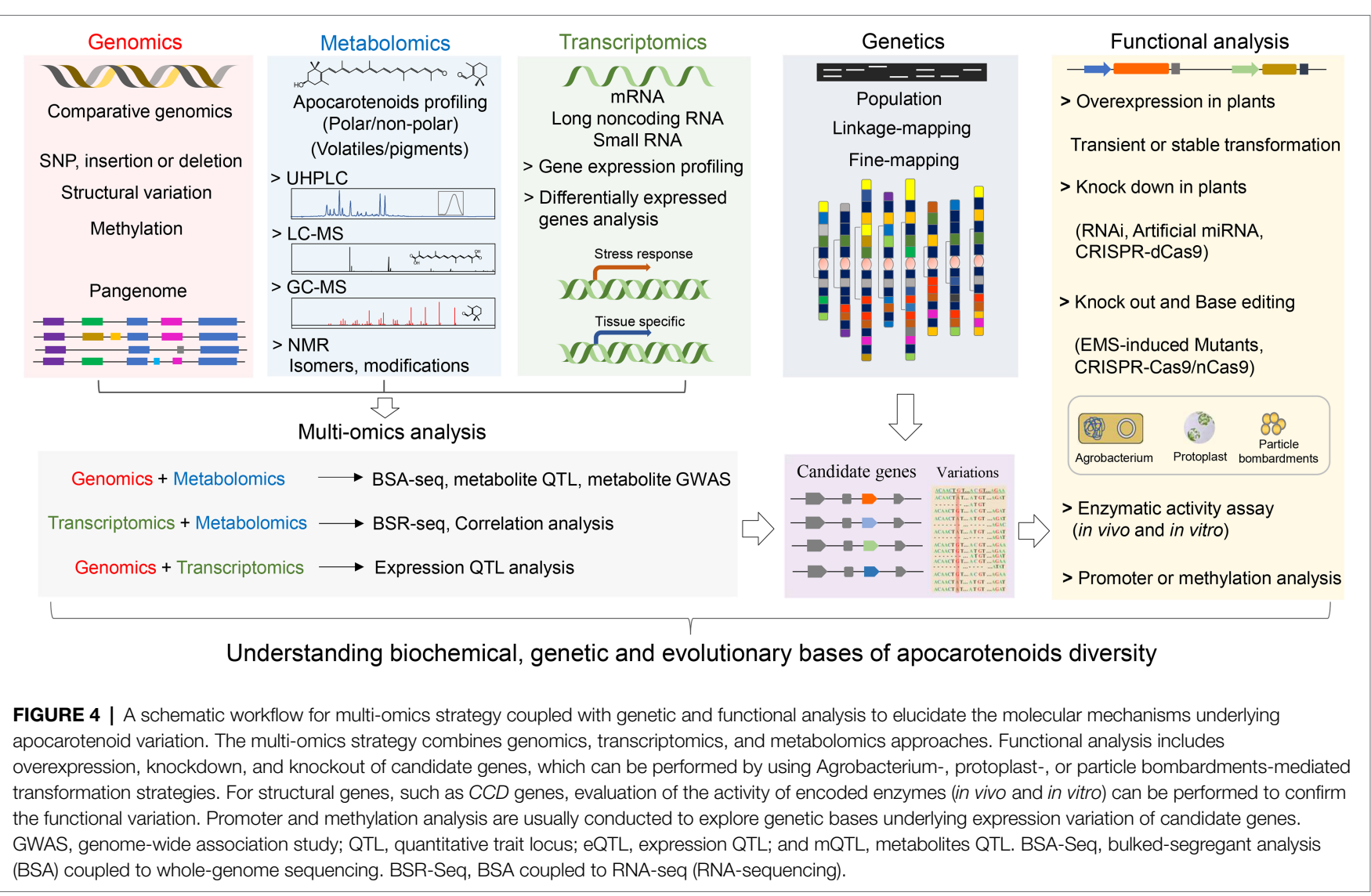

pigments, i.e., $\beta$-citraurin and $\beta$-citraurinene, and their ratio to total carotenoids in citrus peel showed the high correlation with the color index of red-peeled progenies in a $\mathrm{F}_{1}$ pseudo-testcross population derived from a cross between a red-peeled Citrus reticulata and a yellow-peeled Poncirus trifoliata (Zheng et al., 2019). By using mQTL, eQTL, and BSR-seq analysis, this study showed that a $5^{\prime}$ cis-regulatory mutation of Citrus CCD $4 b$ $(C i t C C D 4 b)$ is a major genetic determinant of natural variation in the accumulation of the $C_{30}$ pigments $\beta$-citraurin and $\beta$-citraurinene. This study demonstrated that CitCCD4b enzyme is involved in the biosynthesis of $\beta$-citraurinene, which indicates a novel reaction in modification of aldehyde group of $\beta$-citraurin. The presence of a specific SNP in the MITE of CitCCD $4 b$ promoter is strongly correlated with the high expression level of CitCCD $4 b$ among progenies of pseudo-testcross population as well as within 115 different citrus accessions, resulting in the high-accumulation of $\mathrm{C}_{30}$ apocarotenoid pigments responsible for the red coloration of citrus peel. A recent study also found that a red-peeled mutant of "Huyou" (Citrus changshanensis) contains much higher content of the $\mathrm{C}_{30}$ apocarotenoid $\beta$-citraurin, accompanied by 100 times higher expression level of CitCCD $4 b$, compared to yellow-peeled ordinary "Huyou" (Luan et al., 2020b), which also correlated with the above-mentioned putative enhancer SNP in the CitCCD $4 b$ promoter (Zheng et al., 2019; Luan et al., 2020a). Very recently, it was found that the higher transcript level of CitCCD $4 b$ in red peel of F1 hybrids also accompanied the higher content of the two $\mathrm{C}_{10}$ apocarotenoid volatiles, $\beta$-CC and 3-OH- $\beta$-cyclocitral, in comparison to that in the yellow peel of three other progenies (Zheng et al., 2021). This result suggests a dual role of CitCCD4b expression variation in the natural variation of both apocarotenoid pigments and volatiles among different citrus species and in fruit pigmentation and aroma formation.

However, in other crops, such as peach and Brassica species, mutations in coding sequence of CCD4 enzyme enhanced the carotenoid pigments in tissues/organs, due to the capability of this enzyme in cleaving colorful carotenoids into $\mathrm{C}_{27}$ apocarotenoids that are catabolized into colorless smaller metabolites (Falchi et al., 2013; Mi and Al-Babili, 2019). Recent Studies demonstrated that CRISPR/Cas9-mediated disruption of CCD4 gene in Ipomoea nil and carrot resulted in a remarkable enhancement of carotenoids and led to pale-yellow phenotype in petal of $I$. nil and yellow phenotype in the taproot of a white-colored carrot variety, respectively (Watanabe et al., 2018; Li et al., 2021). The corresponding CCD4 enzymes have also been reported to be responsible for the formation of apocarotenoid volatiles in tissues/organs, such as in peach fruit (Brandi et al., 2011). The crucial step of future research is to determine whether the natural genetic variation of such $C C D 4 \mathrm{~s}$ is also linked to the diversity of apocarotenoid volatiles among different accessions, which may be utilized in molecular breeding for improvement of fruit/vegetable flavor.

Apart from apocarotenoid pigments and volatiles, apocarotenoidderived hormones also show natural variation among different accessions. For example, at low water potential, Arabidopsis accession "Shahdara" (Sha) showed less accumulation of ABA 
than that in "Columbia" (Col) or "Landsberg erecta" (Ler). Genetic analysis of Ler $\times$ Sha recombinant inbred lines (RILs) and complementation experiments demonstrated that $N C E D 3$ variation is responsible for the difference in $\mathrm{ABA}$ accumulation between Sha and Ler accession. Further sequence analysis, site-directed mutagenesis and structural observations revealed that nonsynonymous substitutions of NCED3 coding sequence found in Sha are associated with decreased ABA accumulation and altered NCED3 post-translational processing, which may affect NCED3 activity (Kalladan et al., 2019). The rice cultivars "Bala" and "Azucena" showed large difference in SL biosynthesis that is responsible for their degree of tillering and distinct susceptibility to Striga infection (Cardoso et al., 2014). By using a "Bala"× "Azucena" F6 RIL population, Cardoso et al. (2014) identified a major QTL, a rearrangement of a 51 - to 59-kbp stretch between 28.9 and $29.0 \mathrm{Mbp}$ of chromosome 1, which explains most of the variation in the SL contents of rice exudates. This genomic rearrangement leads to deletion of two AtMAX1 orthologs and is associated with low SL contents in 367 rice cultivars.

\section{CONCLUDING REMARKS AND FUTURE OUTLOOK}

Apocarotenoids are not just carotenoid degradation products but play important functions as antioxidants, pharmaceuticals, scents, and pigments, beside their role as precursors of plant hormones, signaling molecules, and growth regulators. In this review, we have briefly introduced the significance of apocarotenoid compounds for human health and their biological functions that are critical for plant development, biotic interactions, and stress response and provided an update on recently identified biosynthetic pathways and their regulation. More and more studies have shown that exogenous application of apocarotenoids is able to affect plant growth and development, as well as both abiotic and biotic stress resistance in many plants, which uncovered their biological function as regulatory metabolites. Nevertheless, the correlation between natural variation in the content of these apocarotenoids and plant phenotypes remains elusive. It is still unknown why apocarotenoids with slightly different structures have different activities and diverse functions in plants and some apocarotenoids, i.e., zaxinone, exerts contradictory effects depending on plant species. We proposed that the exploration of biochemical and genetic reasons of apocarotenoid metabolic diversity/variation could accelerate exploring currently unknown/ unclear bioactive apocarotenoids pathway that could help

\section{REFERENCES}

Ablazov, A., Mi, J., Jamil, M., Jia, K.-P., Wang, J. Y., Feng, Q., et al. (2020). The apocarotenoid zaxinone is a positive regulator of strigolactone and abscisic acid biosynthesis in Arabidopsis roots. Front. Plant Sci. 11:578. doi: 10.3389/fpls. 2020.00578

Abuauf, H., Haider, I., Jia, K.-P., Ablazov, A., Mi, J., Blilou, I., et al. (2018). The Arabidopsis DWARF27 gene encodes an all-trans-/9-cis- $\beta$-carotene isomerase and is induced by auxin, abscisic acid and phosphate deficiency. Plant Sci. 277, 33-42. doi: 10.1016/j.plantsci.2018.06.024 understand their contributions to crops end-phenotypes, as well as their regulation and modifications that are still poorly understood. To date, the progress in determining natural genetic variation mechanisms and its molecular background is limited to only few apocarotenoid volatiles and pigments, while that of other important apocarotenoids, such as crocins, bixin, zaxinone, and anchorene, and apocarotenoid-derived phytohormones, is still poorly characterized. The rapid development of nextgeneration sequencing strategies provides reliable reference genomic sequences that are now available for most crop plants. We anticipate that mass spectrometry apocarotenoids profiling coupled with multi-omics strategies, including quantitative trait locus (QTL) mapping, genome-wide association (GWA), and RNA-seq analysis, will pave the way for uncovering new QTLs for apocarotenoid metabolite traits and identifying their underlying genes in various important crops (Figure 4). Advancement of knowledge in this area will enable to identify DNA markerapocarotenoid traits associations for marker-assisted improvement of crops agronomic traits, such as aroma and color of fruits and flowers, related to apocarotenoid content, but also provide new metabolic engineering and/or CRISPR editing target for improvements of high-value beneficial apocarotenoids in crops in a low-cost, efficient, stable, and environment-friendly manner, to satisfy increasing demands for better crop quality in the future.

\section{AUTHOR CONTRIBUTIONS}

$\mathrm{XZ}$ organized and drafted the manuscript with substantial input from YY and designed and prepared the figures with input from YY. YY and XZ prepared the table. SA-B supervised the writing of the review and was involved in its further editing and revision. All authors contributed to the article and approved the submitted version.

\section{FUNDING}

This work was supported by baseline funding and Competitive Research Grants (CRG 2017 and CRG 2020) given to SA-B from King Abdullah University of Science and Technology (KAUST).

\section{ACKNOWLEDGMENTS}

We thank Jianing Mi for reading and correcting the manuscript. 
synthesis of crocetin in spring crocuses and saffron is a plastidial enzyme. New Phytol. 209, 650-663. doi: 10.1111/nph.13609

Al-Babili, S., and Bouwmeester, H. J. (2015). Strigolactones, a novel carotenoidderived plant hormone. Annu. Rev. Plant Biol. 66, 161-186. doi: 10.1146/ annurev-arplant-043014-114759

Alder, A., Jamil, M., Marzorati, M., Bruno, M., Vermathen, M., Bigler, P., et al. (2012). The path from $\beta$-carotene to carlactone, a strigolactone-like plant hormone. Science 335, 1348-1351. doi: 10.1126/science.1218094

Ashraf, N., Jain, D., and Vishwakarma, R. A. (2015). Identification, cloning and characterization of an ultrapetala transcription factor CsULT1 from crocus: a novel regulator of apocarotenoid biosynthesis. BMC Plant Biol. 15:25. doi: 10.1186/s12870-015-0423-7

Auldridge, M. E., Block, A., Vogel, J. T., Dabney-Smith, C., Mila, I., Bouzayen, M., et al. (2006). Characterization of three members of the Arabidopsis carotenoid cleavage dioxygenase family demonstrates the divergent roles of this multifunctional enzyme family. Plant J. 45, 982-993. doi: 10.1111/j.1365-313X.2006.02666.x

Baz, L., Mori, N., Mi, J., Jamil, M., Kountche, B. A., Guo, X., et al. (2018). 3-Hydroxycarlactone, a novel product of the strigolactone biosynthesis core pathway. Mol. Plant 11, 1312-1314. doi: 10.1016/j.molp.2018.06.008

Bhuiyan, N. H., Friso, G., Rowland, E., Majsec, K., and Van Wijk, K. J. (2016). The plastoglobule-localized metallopeptidase PGM48 is a positive regulator of senescence in Arabidopsis thaliana. Plant Cell 28, 3020-3037. doi: 10.1105/ tpc. 16.00745

Bhuiyan, N. H., and Van Wijk, K. J. (2017). Functions and substrates of plastoglobule-localized metallopeptidase PGM48. Plant Signal. Behav. 12, 3020-3037. doi: 10.1080/15592324.2017.1331197

Bouvier, F., Dogbo, O., and Camara, B. (2003). Biosynthesis of the food and cosmetic plant pigment bixin (annatto). Science 300, 2089-2091. doi: 10.1126/ science. 1085162

Brandi, F., Bar, E., Mourgues, F., Horváth, G., Turcsi, E., Giuliano, G., et al. (2011). Study of 'Redhaven' peach and its white-fleshed mutant suggests a key role of CCD4 carotenoid dioxygenase in carotenoid and norisoprenoid volatile metabolism. BMC Plant Biol. 11, 1-14. doi: 10.1186/1471-2229-11-24

Bruno, M., and Al-Babili, S. (2016). On the substrate specificity of the rice strigolactone biosynthesis enzyme DWARF27. Planta 243, 1429-1440. doi: 10.1007/s00425-016-2487-5

Bruno, M., Hofmann, M., Vermathen, M., Alder, A., Beyer, P., and Al-Babili, S. (2014). On the substrate-and stereospecificity of the plant carotenoid cleavage dioxygenase 7. FEBS Lett. 588, 1802-1807. doi: 10.1016/j.febslet.2014.03.041

Bruno, M., Koschmieder, J., Wuest, F., Schaub, P., Fehling-Kaschek, M., Timmer, J., et al. (2016). Enzymatic study on AtCCD4 and AtCCD7 and their potential to form acyclic regulatory metabolites. J. Exp. Biol. 67, 5993-6005. doi: 10.1093/jxb/erw356

Bruno, M., Vermathen, M., Alder, A., Wüst, F., Schaub, P., Van Der Steen, R., et al. (2017). Insights into the formation of carlactone from in-depth analysis of the CCD 8-catalyzed reactions. FEBS Lett. 591, 792-800. doi: $10.1002 / 1873-3468.12593$

Bukhari, S. I., Manzoor, M., and Dhar, M. (2018). A comprehensive review of the pharmacological potential of Crocus sativus and its bioactive apocarotenoids. Biomed. Pharmacother. 98, 733-745. doi: 10.1016/j.biopha.2017.12.090

Cáceres, L., Lakshminarayan, S., Yeung, K.-C., Mcgarvey, B., Hannoufa, A., Sumarah, M., et al. (2016). Repellent and attractive effects of $\alpha-, \beta$-, and dihydro- $\beta$-ionone to generalist and specialist herbivores. J. Chem. Ecol. 42, 107-117. doi: 10.1007/s10886-016-0669-z

Campbell, R., Ducreux, L. J., Morris, W. L., Morris, J. A., Suttle, J. C., Ramsay, G., et al. (2010). The metabolic and developmental roles of carotenoid cleavage dioxygenase 4 from potato. Plant Physiol. 154, 656-664. doi: 10.1104/ pp. 110.158733

Cardoso, C., Zhang, Y., Jamil, M., Hepworth, J., Charnikhova, T., Dimkpa, S. O., et al. (2014). Natural variation of rice strigolactone biosynthesis is associated with the deletion of two MAX1 orthologs. Proc. Natl. Acad. Sci. U. S. A. 111, 2379-2384. doi: 10.1073/pnas.1317360111

Carrera, A., Echenique, V., Zhang, W., Helguera, M., Manthey, F., Schrager, A., et al. (2007). A deletion at the Lpx-B1 locus is associated with low lipoxygenase activity and improved pasta color in durum wheat (Triticum turgidum ssp. durum). J. Cereal Sci. 45, 67-77. doi: 10.1016/j.jcs.2006.07.001

Chayut, N., Yuan, H., Ohali, S., Meir, A., Sa’ar, U., Tzuri, G., et al. (2017). Distinct mechanisms of the ORANGE protein in controlling carotenoid flux. Plant Physiol. 173, 376-389. doi: 10.1104/pp.16.01256
Chen, K., Li, G. J., Bressan, R. A., Song, C. P., Zhu, J. K., and Zhao, Y. (2020). Abscisic acid dynamics, signaling, and functions in plants. J. Integr. Plant Biol. 62, 25-54. doi: 10.1111/jipb.12899

Chiou, C.-Y., Pan, H.-A., Chuang, Y.-N., and Yeh, K.-W. (2010). Differential expression of carotenoid-related genes determines diversified carotenoid coloration in floral tissues of Oncidium cultivars. Planta 232, 937-948. doi: 10.1007/s00425-010-1222-x

Cook, C., Whichard, L. P., Turner, B., Wall, M. E., and Egley, G. H. (1966). Germination of witchweed (Striga lutea lour.): isolation and properties of a potent stimulant. Science 154, 1189-1190. doi: 10.1126/science.154.3753.1189

D'alessandro, S., Mizokami, Y., Legeret, B., and Havaux, M. (2019). The apocarotenoid $\beta$-cyclocitric acid elicits drought tolerance in plants. Iscience 19, 461-473. doi: 10.1016/j.isci.2019.08.003

Decker, E. L., Alder, A., Hunn, S., Ferguson, J., Lehtonen, M. T., Scheler, B., et al. (2017). Strigolactone biosynthesis is evolutionarily conserved, regulated by phosphate starvation and contributes to resistance against phytopathogenic fungi in a moss, Physcomitrella patens. New Phytol. 216, 455-468. doi: 10.1111/nph.14506

DellaPenna, D., and Pogson, B. J. (2006). Vitamin synthesis in plants: tocopherols and carotenoids. Annu. Rev. Plant Biol. 57, 711-738. doi: 10.1146/annurev. arplant.56.032604.144301

Demurtas, O. C., Frusciante, S., Ferrante, P., Diretto, G., Azad, N. H., Pietrella, M., et al. (2018). Candidate enzymes for saffron crocin biosynthesis are localized in multiple cellular compartments. Plant Physiol. 177, 990-1006. doi: 10.1104/ pp. 17.01815

Dickinson, A. J., Lehner, K., Mi, J., Jia, K.-P., Mijar, M., Dinneny, J., et al. (2019). $\beta$-Cyclocitral is a conserved root growth regulator. Proc. Natl. Acad. Sci. U. S. A. 116, 10563-10567. doi: 10.1073/pnas.1821445116

Diretto, G., Ahrazem, O., Rubio-Moraga, Á., Fiore, A., Sevi, F., Argandoña, J., et al. (2019). UGT709G1: a novel uridine diphosphate glycosyltransferase involved in the biosynthesis of picrocrocin, the precursor of safranal in saffron (Crocus sativus). New Phytol. 224, 725-740. doi: 10.1111/nph.16079

Falchi, R., Vendramin, E., Zanon, L., Scalabrin, S., Cipriani, G., Verde, I., et al. (2013). Three distinct mutational mechanisms acting on a single gene underpin the origin of yellow flesh in peach. Plant J. 76, 175-187. doi: $10.1111 /$ tpj. 12283

Fang, Q., Li, Y., Liu, B., Meng, X., Yang, Z., Yang, S., et al. (2020). Cloning and functional characterization of a carotenoid cleavage dioxygenase 2 gene in safranal and crocin biosynthesis from Freesia hybrida. Plant Physiol. Biochem. 154, 439-450. doi: 10.1016/j.plaphy.2020.06.035

Felemban, A., Braguy, J., Zurbriggen, M. D., and Al-Babili, S. (2019). Apocarotenoids involved in plant development and stress response. Front. Plant Sci. 10:1168. doi: 10.3389/fpls.2019.01168

Fiedor, J., Fiedor, L., Haeßner, R., and Scheer, H. (2005). Cyclic endoperoxides of $\beta$-carotene, potential pro-oxidants, as products of chemical quenching of singlet oxygen. Biochim. Biophys. Acta, Bioenerg. 1709, 1-4. doi: 10.1016/j. bbabio.2005.05.008

Fiorilli, V., Wang, J. Y., Bonfante, P., Lanfranco, L., and Al-Babili, S. (2019). Apocarotenoids: old and new mediators of the arbuscular mycorrhizal symbiosis. Front. Plant Sci. 10:1186. doi: 10.3389/fpls.2019.01186

Fraser, P. D., and Bramley, P. M. (2004). The biosynthesis and nutritional uses of carotenoids. Prog. Lipid Res. 43, 228-265. doi: 10.1016/j.plipres.2003.10.002

Frusciante, S., Diretto, G., Bruno, M., Ferrante, P., Pietrella, M., Prado-Cabrero, A., et al. (2014). Novel carotenoid cleavage dioxygenase catalyzes the first dedicated step in saffron crocin biosynthesis. Proc. Natl. Acad. Sci. U. S. A. 111, 12246-12251. doi: 10.1073/pnas.1404629111

Gao, L., Gonda, I., Sun, H., Ma, Q., Bao, K., Tieman, D. M., et al. (2019). The tomato pan-genome uncovers new genes and a rare allele regulating fruit flavor. Nat. Genet. 51, 1044-1051. doi: 10.1038/s41588-019-0410-2

García-Limones, C., SchnäBele, K., Blanco-Portales, R., Luz Bellido, M., Caballero, J. L., Schwab, W., et al. (2008). Functional characterization of FaCCD1: a carotenoid cleavage dioxygenase from strawberry involved in lutein degradation during fruit ripening. J. Agric. Food Chem. 56, 9277-9285. doi: $10.1021 / \mathrm{jf} 801096 \mathrm{t}$

Gayen, D., Ali, N., Sarkar, S. N., Datta, S. K., and Datta, K. (2015). Downregulation of lipoxygenase gene reduces degradation of carotenoids of golden rice during storage. Planta 242, 353-363. doi: 10.1007/s00425-015-2314-4

Giuliano, G. (2014). Plant carotenoids: genomics meets multi-gene engineering. Curr. Opin. Plant Biol. 19, 111-117. doi: 10.1016/j.pbi.2014.05.006 
Giuliano, G. (2017). Provitamin A biofortification of crop plants: a gold rush with many miners. Curr. Opin. Plant Biol. 44, 169-180. doi: 10.1016/j. copbio.2017.02.001

Giuliano, G., Al-Babili, S., and Von Lintig, J. (2003a). Carotenoid oxygenases: cleave it or leave it. Trends Plant Sci. 8, 145-149. doi: 10.1016/S1360-1385(03)00053-0

Giuliano, G., Rosati, C., and Bramley, P. M. (2003b). To dye or not to dye: biochemistry of annatto unveiled. Trends Biotechnol. 21, 513-516. doi: 10.1016/j.tibtech.2003.10.001

Goff, S. A., and Klee, H. J. (2006). Plant volatile compounds: sensory cues for health and nutritional value? Science 311, 815-819. doi: 10.1126/ science. 1112614

González-Guzmán, M., Apostolova, N., Bellés, J. M., Barrero, J. M., Piqueras, P., Ponce, M. R., et al. (2002). The short-chain alcohol dehydrogenase ABA2 catalyzes the conversion of xanthoxin to abscisic aldehyde. Plant Cell 14, 1833-1846. doi: $10.1105 /$ tpc.002477

Gonzalez-Jorge, S., Ha, S. H., Magallanes-Lundback, M., Gilliland, L. U., Zhou, A., Lipka, A. E., et al. (2013). Carotenoid cleavage dioxygenase 4 is a negative regulator of $\beta$-carotene content in Arabidopsis seeds. Plant Cell 25, 4812-4826. doi: $10.1105 /$ tpc.113.119677

González-Mas, M. C., Rambla, J. L., Alamar, M. C., Gutiérrez, A., and Granell, A. (2011). Comparative analysis of the volatile fraction of fruit juice from different citrus species. PLoS One 6:e22016. doi: 10.1371/journal.pone.0022016

Gutheil, W. G., Reed, G., Ray, A., Anant, S., and Dhar, A. (2012). Crocetin: an agent derived from saffron for prevention and therapy for cancer. Curr. Pharm. Biotechnol. 13, 173-179. doi: 10.2174/138920112798868566

Han, F., Cui, H., Zhang, B., Liu, X., Yang, L., Zhuang, M., et al. (2019a). Map-based cloning and characterization of BoCCD4, a gene responsible for white/yellow petal color in B. oleracea. BMC Genomics 20:242. doi: 10.1186/ s12864-019-5596-2

Han, Y., Wang, H., Wang, X., Li, K., Dong, M., Li, Y., et al. (2019b). Mechanism of floral scent production in Osmanthus fragrans and the production and regulation of its key floral constituents, $\beta$-ionone and linalool. Hortic. Res. 6:106. doi: 10.1038/s41438-019-0189-4

Han, Y., Wu, M., Cao, L., Yuan, W., Dong, M., Wang, X., et al. (2016). Characterization of OfWRKY3, a transcription factor that positively regulates the carotenoid cleavage dioxygenase gene OfCCD4 in Osmanthus fragrans. Plant Mol. Biol. 91, 485-496. doi: 10.1007/s11103-016-0483-6

Harrison, E. H. (2005). Mechanisms of digestion and absorption of dietary vitamin A. Annu. Rev. Nutr. 25, 87-103. doi: 10.1146/annurev.nutr.25.050304.092614

Hasegawa, T., Bando, A., Tsuchiya, K., Abe, S., Okamoto, M., Kirima, K., et al. (2004). Enzymatic and nonenzymatic formation of reactive oxygen species from 6-anilino-5, 8-quinolinequinone. Biophys Acta Gen Subj. 1670, 19-27. doi: 10.1016/j.bbagen.2003.10.008

Hashimoto, H., Uragami, C., and Cogdell, R. J. (2016). Carotenoids and photosynthesis. Carotenoid. Nat. 79, 111-139. doi: 10.1007/978-3-319-39126-7_4

Havaux, M. (2020). $\beta$-Cyclocitral and derivatives: emerging molecular signals serving multiple biological functions. Plant Physiol. Biochem. 155, 35-41. doi: $10.1016 /$ j.plaphy.2020.07.032

Hirschberg, J. (2001). Carotenoid biosynthesis in flowering plants. Curr. Opin. Plant Biol. 4, 210-218. doi: 10.1016/S1369-5266(00)00163-1

Hou, X., Rivers, J., León, P., Mcquinn, R. P., and Pogson, B. J. (2016). Synthesis and function of apocarotenoid signals in plants. Trends Plant Sci. 21, 792-803. doi: $10.1016 /$ j.tplants.2016.06.001

Huang, F.-C., Molnár, P., and Schwab, W. (2009). Cloning and functional characterization of carotenoid cleavage dioxygenase 4 genes. J. Exp. Bot. 60, 3011-3022. doi: 10.1093/jxb/erp137

Ibdah, M., Azulay, Y., Portnoy, V., Wasserman, B., Bar, E., Meir, A., et al. (2006). Functional characterization of CmCCD1, a carotenoid cleavage dioxygenase from melon. Phytochemistry 67, 1579-1589. doi: 10.1016/j. phytochem.2006.02.009

Ilg, A., Beyer, P., and Al-Babili, S. (2009). Characterization of the rice carotenoid cleavage dioxygenase 1 reveals a novel route for geranial biosynthesis. FEBS J. 276, 736-747. doi: 10.1111/j.1742-4658.2008.06820.x

Ilg, A., Bruno, M., Beyer, P., and Al-Babili, S. (2014). Tomato carotenoid cleavage dioxygenases $1 \mathrm{~A}$ and $1 \mathrm{~B}$ : relaxed double bond specificity leads to a plenitude of dialdehydes, mono-apocarotenoids and isoprenoid volatiles. FEBS Open Bio. 4, 584-593. doi: 10.1016/j.fob.2014.06.005

Ilg, A., Yu, Q., Schaub, P., Beyer, P., and Al-Babili, S. (2010). Overexpression of the rice carotenoid cleavage dioxygenase 1 gene in Golden Rice endosperm suggests apocarotenoids as substrates in planta. Planta 232, 691-699. doi: 10.1007/s00425-010-1205-y

Jamil, M., Kountche, B. A., and Al-Babili, S. (2021). Current progress in Striga management. Plant Physiol. 185, 1339-1352. doi: 10.1093/plphys/kiab040

Jarén-Galán, M., and Mínguez-Mosquera, M. I. (1999). Effect of pepper lipoxygenase activity and its linked reactions on pigments of the pepper fruit. J. Agric. Food Chem. 47, 4532-4536. doi: 10.1021/j99900682

Jia, K.-P., Baz, L., and Al-Babili, S. (2018). From carotenoids to strigolactones. J. Exp. Biol. 69, 2189-2204. doi: 10.1093/jxb/erx476

Jia, K.-P., Dickinson, A. J., Mi, J., Cui, G., Xiao, T. T., Kharbatia, N. M., et al. (2019). Anchorene is a carotenoid-derived regulatory metabolite required for anchor root formation in Arabidopsis. Sci. Adv. 5:eaaw6787. doi: 10.1126/ sciadv.aaw6787

Jia, K.-P., Mi, J., Ablazov, A., Ali, S., Yang, Y., Balakrishna, A., et al. (2021a). Iso-anchorene is an endogenous metabolite that inhibits primary root growth in Arabidopsis. Plant J. 107, 54-66. doi: 10.1111/tpj.15271

Jia, K.-P., Mi, J., Ali, S., Ohyanagi, H., Moreno, J.C., Ablazov, A., et al. (2021b). An alternative, zeaxanthin epoxidase-independent abscisic acid biosynthetic pathway in plants. Mol. Plant doi: 10.1016/j.molp.2021.09.008 [Epub ahead of print].

Jian, W., Cao, H., Yuan, S., Liu, Y., Lu, J., Lu, W., et al. (2019). SIMYB75, an MYB-type transcription factor, promotes anthocyanin accumulation and enhances volatile aroma production in tomato fruits. Hortic. Res. 6:22. doi: 10.1038/s41438-018-0098-y

Jing, G., Li, T., Qu, H., Yun, Z., Jia, Y., Zheng, X., et al. (2015). Carotenoids and volatile profiles of yellow-and red-fleshed papaya fruit in relation to the expression of carotenoid cleavage dioxygenase genes. Postharvest Biol. Technol. 109, 114-119. doi: 10.1016/j.postharvbio.2015.06.006

Kalladan, R., Lasky, J. R., Sharma, S., Kumar, M. N., Juenger, T. E., Des Marais, D. L., et al. (2019). Natural variation in 9-cis-epoxycartenoid dioxygenase 3 and ABA accumulation. Plant Physiol. 179, 1620-1631. doi: 10.1104/pp.18.01185

Kong, C.-H., Zhang, S.-Z., Li, Y.-H., Xia, Z.-C., Yang, X.-F., Meiners, S. J., et al. (2018). Plant neighbor detection and allelochemical response are driven by root-secreted signaling chemicals. Nat. Commun. 9:3867. doi: 10.1038/ s41467-018-06429-1

Lanfranco, L., Fiorilli, V., Venice, F., and Bonfante, P. (2018). Strigolactones cross the kingdoms: plants, fungi, and bacteria in the arbuscular mycorrhizal symbiosis. J. Exp. Biol. 69, 2175-2188. doi: 10.1093/jxb/erx432

Lashbrooke, J. G., Young, P. R., Dockrall, S. J., Vasanth, K., and Vivier, M. A. (2013). Functional characterisation of three members of the Vitis vinifera L. carotenoid cleavage dioxygenase gene family. BMC Plant Biol. 13:156. doi: 10.1186/1471-2229-13-156

Li, T., Deng, Y.J., Liu, J.X., Duan, A.Q., Liu, H., and Xiong, A.S. (2021). DcCCD4 catalyzes the degradation of $\alpha$-carotene and $\beta$-carotene to affect carotenoid accumulation and taproot color in carrot. Plant J. doi: 10.1111/ tpj.15524 [Epub ahead of print].

Li, L.-L., Zhao, H.-H., and Kong, C.-H. (2020). (-)-Loliolide, the most ubiquitous lactone, is involved in barnyardgrass-induced rice allelopathy. J. Exp. Bot. 71, 1540-1550. doi: 10.1093/jxb/erz497

Liang, M.-H., He, Y.-J., Liu, D.-M., and Jiang, J.-G. (2021). Regulation of carotenoid degradation and production of apocarotenoids in natural and engineered organisms. Crit. Rev. Biotechnol. 41, 531-534. doi: 10.1080/07388551.2021.1873242

Liu, F., Zhang, H., Ding, L., Soppe, W. J., and Xiang, Y. (2020). Reversal of rdo51, a homolog of rice seed dormancy4, interacts with bHLH57 and controls ABA biosynthesis and seed dormancy in Arabidopsis. Plant Cell 32, 1933-1948. doi: 10.1105/tpc.20.00026

Lu, S., Zhang, Y., Zhu, K., Yang, W., Ye, J., Chai, L., et al. (2018). The citrus transcription factor CsMADS6 modulates carotenoid metabolism by directly regulating carotenogenic genes. Plant Physiol. 176, 2657-2676. doi: 10.1104/ pp.17.01830

Luan, Y., Fu, X., Lu, P., Grierson, D., and Xu, C. (2020a). Molecular mechanisms determining the differential accumulation of carotenoids in plant species and varieties. Crit. Rev. Plant Sci. 39, 125-139. doi: 10.1080/07352689.2020.1768350

Luan, Y., Wang, S., Wang, R., and Xu, C. (2020b). Accumulation of red apocarotenoid $\beta$-citraurin in peel of a spontaneous mutant of huyou (citrus changshanensis) and the effects of storage temperature and ethylene application. Food Chem. 309:125705. doi: 10.1016/j.foodchem.2019.125705

Ma, G., Zhang, L., Matsuta, A., Matsutani, K., Yamawaki, K., Yahata, M., et al. (2013). Enzymatic formation of $\beta$-citraurin from $\beta$-cryptoxanthin and 
zeaxanthin by carotenoid cleavage dioxygenase 4 in the flavedo of citrus fruit. Plant Physiol. 163, 682-695. doi: 10.1104/pp.113.223297

Martí, M., Diretto, G., Aragonés, V., Frusciante, S., Ahrazem, O., Gómez-Gómez, L., et al. (2020). Efficient production of saffron crocins and picrocrocin in Nicotiana benthamiana using a virus-driven system. Metab. Eng. 61, 238-250. doi: 10.1016/j.ymben.2020.06.009

Mathieu, S., Terrier, N., Procureur, J. M., Bigey, F., and Günata, Z. (2005). A carotenoid cleavage dioxygenase from Vitis vinifera L.: functional characterization and expression during grape berry development in relation to C13-norisoprenoid accumulation. J. Exp. Bot. 56, 2721-2731. doi: 10.1093/jxb/eri265

Meng, N., Wei, Y., Gao, Y., Yu, K., Cheng, J., Li, X.-Y., et al. (2020). Characterization of transcriptional expression and regulation of carotenoid cleavage dioxygenase $4 b$ in grapes. Front. Plant Sci. 11:483. doi: 10.3389/fpls.2020.00483

Mi, J., and Al-Babili, S. (2019). To color or to decolor: that is the question. Mol. Plant 12, 1173-1175. doi: 10.1016/j.molp.2019.07.007

Mi, J., Jia, K.-P., Balakrishna, A., Feng, Q., and Al-Babili, S. (2019). A highly sensitive SPE derivatization-UHPLC-MS approach for quantitative profiling of carotenoid-derived dialdehydes from vegetables. J. Agric. Food Chem. 67, 5899-5907. doi: 10.1021/acs.jafc.9b01749

Mitra, S., Estrada-Tejedor, R., Volke, D. C., Phillips, M. A., Gershenzon, J., and Wright, L. P. (2021). Negative regulation of plastidial isoprenoid pathway by herbivore-induced $\beta$-cyclocitral in Arabidopsis thaliana. Proc. Natl. Acad. Sci. U. S. A. 118:e2008747118. doi: 10.1073/pnas.2008747118

Moise, A. R., Al-Babili, S., and Wurtzel, E. T. (2014). Mechanistic aspects of carotenoid biosynthesis. Chem. Rev. 114, 164-193. doi: 10.1021/cr400106y

Moreno, J. C., Mi, J., Alagoz, Y., and Al-Babili, S. (2021). Plant apocarotenoids: from retrograde signaling to interspecific communication. Plant J. 105, 351-375. doi: 10.1111/tpj.15102

Murata, M., Kobayashi, T., and Seo, S. (2020). $\alpha$-Ionone, an apocarotenoid, induces plant resistance to western flower thrips, Frankliniella occidentalis, independently of jasmonic acid. Molecules 25:17. doi: 10.3390/molecules25010017

Murata, M., Nakai, Y., Kawazu, K., Ishizaka, M., Kajiwara, H., Abe, H., et al. (2019). Loliolide, a carotenoid metabolite, is a potential endogenous inducer of herbivore resistance. Plant Physiol. 179, 1822-1833. doi: 10.1104/pp.18.00837

Nisar, N., Li, L., Lu, S., Khin, N. C., and Pogson, B. J. (2015). Carotenoid metabolism in plants. Mol. Plant 8, 68-82. doi: 10.1016/j.molp.2014.12.007

Ohmiya, A., Kishimoto, S., Aida, R., Yoshioka, S., and Sumitomo, K. (2006). Carotenoid cleavage dioxygenase (CmCCD4a) contributes to white color formation in chrysanthemum petals. Plant Physiol. 142, 1193-1201. doi: 10.1104/pp.106.087130

Pacheco, S. D. G., Gasparin, A. T., Jesus, C. H. A., Sotomaior, B. B., Ventura, A. C. S. S. B., Redivo, D. D. B., et al. (2019). Antinociceptive and anti-inflammatory effects of bixin, a carotenoid extracted from the seeds of Bixa orellana. Planta Med. 85, 1216-1224. doi: 10.1055/a-1008-1238

Park, S.-C., Kang, L., Park, W. S., Ahn, M.-J., Kwak, S.-S., and Kim, H. S. (2020). Carotenoid cleavage dioxygenase 4 (CCD4) cleaves $\beta$-carotene and interacts with IbOr in sweetpotato. Plant Biotechnol. Rep. 14, 737-742. doi: 10.1007/s11816-020-00649-y

Park, S., Kim, H. S., Jung, Y. J., Kim, S. H., Ji, C. Y., Wang, Z., et al. (2016). Orange protein has a role in phytoene synthase stabilization in sweetpotato. Sci. Rep. 6, 1-12. doi: 10.1038/srep33563

Ramel, F., Birtic, S., Ginies, C., Soubigou-Taconnat, L., Triantaphylides, C., and Havaux, M. (2012). Carotenoid oxidation products are stress signals that mediate gene responses to singlet oxygen in plants. Proc. Natl. Acad. Sci. U. S. A. 109, 5535-5540. doi: 10.1073/pnas.1115982109

Reidel, R. V. B., Melai, B., Cioni, P., Flamini, G., and Pistelli, L. (2016). Aroma profile of Rubus ulmifolius flowers and fruits during different ontogenetic phases. Chem. Biodivers. 13, 1776-1784. doi: 10.1002/cbdv.201600170

Rezaee, R., and Hosseinzadeh, H. (2013). Safranal: from an aromatic natural product to a rewarding pharmacological agent. Iran. J. Basic Med. Sci. 16, 12-26.

Rodrigo, M. J., Alquezar, B., Alos, E., Medina, V., Carmona, L., Bruno, M., et al. (2013). A novel carotenoid cleavage activity involved in the biosynthesis of citrus fruit-specific apocarotenoid pigments. J. Exp. Bot. 64, 4461-4478. doi: $10.1093 / \mathrm{jxb} / \mathrm{ert} 260$

Rodriguez-Concepcion, M., Avalos, J., Bonet, M. L., Boronat, A., Gomez-Gomez, L., Hornero-Mendez, D., et al. (2018). A global perspective on carotenoids: metabolism, biotechnology, and benefits for nutrition and health. Prog. Lipid Res. 70, 62-93. doi: 10.1016/j.plipres.2018.04.004
Schwartz, S. H., Qin, X., and Zeevaart, J. A. (2001). Characterization of a novel carotenoid cleavage dioxygenase from plants. J. Biol. Chem. 276, 25208-25211. doi: 10.1074/jbc.M102146200

Schwartz, S. H., Tan, B. C., Gage, D. A., Zeevaart, J. A., and Mccarty, D. R. (1997). Specific oxidative cleavage of carotenoids by VP14 of maize. Science 276, 1872-1874. doi: 10.1126/science.276.5320.1872

Seo, M., Peeters, A. J., Koiwai, H., Oritani, T., Marion-Poll, A., Zeevaart, J. A., et al. (2000). The Arabidopsis aldehyde oxidase 3 (AAO3) gene product catalyzes the final step in abscisic acid biosynthesis in leaves. Proc. Natl. Acad. Sci. U. S. A. 97, 12908-12913. doi: 10.1073/pnas.220426197

Shi, J., Cao, C., Xu, J., and Zhou, C. (2020). Research advances on biosynthesis, regulation, and biological activities of apocarotenoid aroma in horticultural plants. J. Chemother. 2020, 1-11. doi: 10.1155/2020/2526956

Simkin, A. J., Schwartz, S. H., Auldridge, M., Taylor, M. G., and Klee, H. J. (2004a). The tomato carotenoid cleavage dioxygenase 1 genes contribute to the formation of the flavor volatiles $\beta$-ionone, pseudoionone, and geranylacetone. Plant J. 40, 882-892. doi: 10.1111/j.1365-313X.2004.02263.x

Simkin, A. J., Underwood, B. A., Auldridge, M., Loucas, H. M., Shibuya, K., Schmelz, E., et al. (2004b). Circadian regulation of the PhCCD1 carotenoid cleavage dioxygenase controls emission of $\beta$-ionone, a fragrance volatile of petunia flowers. Plant Physiol. 136, 3504-3514. doi: 10.1104/pp.104.049718

Stratton, S. P., Schaefer, W. H., and Liebler, D. C. (1993). Isolation and identification of singlet oxygen oxidation products of $\beta$-carotene. Chem. Res. Toxicol. 6, 542-547. doi: 10.1021/tx00034a024

Sui, X., Kiser, P. D., Von Lintig, J., and Palczewski, K. (2013). Structural basis of carotenoid cleavage: from bacteria to mammals. Arch. Biochem. Biophys. 539, 203-213. doi: 10.1016/j.abb.2013.06.012

Tan, B.-C., Joseph, L. M., Deng, W.-T., Liu, L., Li, Q.-B., Cline, K., et al. (2003). Molecular characterization of the Arabidopsis 9-cis epoxycarotenoid dioxygenase gene family. Plant J. 35, 44-56. doi: 10.1046/j.1365-313X.2003.01786.x

Tieman, D., Zhu, G., Resende, M. F., Lin, T., Nguyen, C., Bies, D., et al. (2017). A chemical genetic roadmap to improved tomato flavor. Science 355, 391-394. doi: 10.1126/science.aal1556

Vogel, J. T., Tan, B.-C., Mccarty, D. R., and Klee, H. J. (2008). The carotenoid cleavage dioxygenase 1 enzyme has broad substrate specificity, cleaving multiple carotenoids at two different bond positions. J. Biol. Chem. 283, 11364-11373. doi: 10.1074/jbc.M710106200

Von Lintig, J. (2010). Colors with functions: elucidating the biochemical and molecular basis of carotenoid metabolism. Annu. Rev. Nutr. 30, 35-56. doi: 10.1146/annurev-nutr-080508-141027

Walter, M. H., and Strack, D. (2011). Carotenoids and their cleavage products: biosynthesis and functions. Nat. Prod. Rep. 28, 663-692. doi: 10.1039/c0np00036a

Wang, J. Y., Alseekh, S., Xiao, T., Ablazov, A., De Souza, L. P., Fiorilli, V., et al. (2021). Multi-omics approaches explain the growth-promoting effect of the apocarotenoid growth regulator zaxinone in rice. Commun. Biol. 4:1222. doi: 10.1038/s42003-021-02740-8

Wang, J. Y., Haider, I., Jamil, M., Fiorilli, V., Saito, Y., Mi, J., et al. (2019a). The apocarotenoid metabolite zaxinone regulates growth and strigolactone biosynthesis in rice. Nat. Commun. 10:810. doi: 10.1038/s41467-019-08461-1

Wang, J. Y., Jamil, M., Lin, P.-Y., Ota, T., Fiorilli, V., Novero, M., et al. (2020a). Efficient mimics for elucidating zaxinone biology and promoting agricultural applications. Mol. Plant 13, 1654-1661. doi: 10.1016/j.molp.2020.08.009

Wang, J. Y., Lin, P.-Y., and Al-Babili, S. (2020b). On the biosynthesis and evolution of apocarotenoid plant growth regulators. Semin. Cell Dev. Biol. 109, 3-11. doi: 10.1016/j.semcdb.2020.07.007

Wang, X., Zeng, W., Ding, Y., Wang, Y., Niu, L., Yao, J.-L., et al. (2019b). PpERF3 positively regulates ABA biosynthesis by activating PpNCED2/3 transcription during fruit ripening in peach. Hortic. Res. 6:19. doi: 10.1038/ s41438-018-0094-2

Watanabe, K., Oda-Yamamizo, C., Sage-Ono, K., Ohmiya, A., and Ono, M. (2018). Alteration of flower colour in Ipomoea nil through CRISPR/Cas9mediated mutagenesis of carotenoid cleavage dioxygenase 4. Transgenic Res. 27, 25-38. doi: 10.1007/s11248-017-0051-0

Watkins, J. L., and Pogson, B. J. (2020). Prospects for carotenoid biofortification targeting retention and catabolism. Trends Plant Sci. 25, 501-512. doi: 10.1016/j.tplants.2019.12.021

Xi, W., Zheng, H., Zhang, Q., and Li, W. (2016). Profiling taste and aroma compound metabolism during apricot fruit development and ripening. Int. J. Mol. Sci. 17:998. doi: 10.3390/ijms17070998 
Xie, X., Yoneyama, K., and Yoneyama, K. (2010). The strigolactone story. Annu. Rev. Phytopathol. 48, 93-117. doi: 10.1146/annurev-phyto-073009-114453

Xu, P., Chen, H., and Cai, W. (2020a). Transcription factor CDF4 promotes leaf senescence and floral organ abscission by regulating abscisic acid and reactive oxygen species pathways in Arabidopsis. EMBO Rep. 21:e48967. doi: $10.15252 / \mathrm{embr} .201948967$

Xu, Z., Pu, X., Gao, R., Demurtas, O. C., Fleck, S. J., Richter, M., et al. (2020b). Tandem gene duplications drive divergent evolution of caffeine and crocin biosynthetic pathways in plants. BMC Biol. 18:63. doi: 10.1186/s12915-020-00795-3

Xu, J., Wang, X., Cao, H., Xu, H., Xu, Q., and Deng, X. (2017). Dynamic changes in methylome and transcriptome patterns in response to methyltransferase inhibitor 5-azacytidine treatment in citrus. DNA Res. 24, 509-522. doi: 10.1093/dnares/dsx021

Yahyaa, M., Bar, E., Dubey, N. K., Meir, A., Davidovich-Rikanati, R., Hirschberg, J., et al. (2013). Formation of norisoprenoid flavor compounds in carrot (Daucus carota L.) roots: characterization of a cyclic-specific carotenoid cleavage dioxygenase 1 gene. J. Agric. Food Chem. 61, 12244-12252. doi: 10.1021/jf404085k

Yamauchi, T., Watanabe, K., Fukazawa, A., Mori, H., Abe, F., Kawaguchi, K., et al. (2014). Ethylene and reactive oxygen species are involved in root aerenchyma formation and adaptation of wheat seedlings to oxygen-deficient conditions. J. Exp. Bot. 65, 261-273. doi: 10.1093/jxb/ert371

Yao, M., Chen, W., Kong, J., Zhang, X., Shi, N., Zhong, S., et al. (2020). Methyltransferase 1 and ripening modulate vivipary during tomato fruit development. Plant Physiol. 183, 1883-1897. doi: 10.1104/pp.20.00499

Yoneyama, K., Akiyama, K., Brewer, P. B., Mori, N., Kawano-Kawada, M., Haruta, S., et al. (2020). Hydroxyl carlactone derivatives are predominant strigolactones in Arabidopsis. Plant Direct 4:e00219. doi: 10.1002/pld3.219

Yu, Q., Plotto, A., Baldwin, E. A., Bai, J., Huang, M., Yu, Y., et al. (2015). Proteomic and metabolomic analyses provide insight into production of volatile and non-volatile flavor components in mandarin hybrid fruit. $B M C$ Plant Biol. 15:76. doi: 10.1186/s12870-015-0466-9

Yuan, H., Zhang, J., Nageswaran, D., and Li, L. (2015). Carotenoid metabolism and regulation in horticultural crops. Hortic. Res. 2:15036. doi: 10.1038/ hortres.2015.36

Yue, Z., Liu, H., and Ma, F. (2015). The malus carotenoid cleavage dioxygenase 7 is involved in stress response and regulated by basic pentacysteine 1 . Sci. Hortic. 192, 264-270. doi: 10.1016/j.scienta.2015.06.027

Zhang, B., Liu, C., Wang, Y., Yao, X., Wang, F., Wu, J., et al. (2015). Disruption of a CAROTENOID CLEAVAGE DIOXYGENASE 4 gene converts flower colour from white to yellow in brassica species. New Phytol. 206, 1513-1526. doi: $10.1111 /$ nph.13335

Zhang, Y., Van Dijk, A. D., Scaffidi, A., Flematti, G. R., Hofmann, M., Charnikhova, T., et al. (2014). Rice cytochrome P450 MAX1 homologs catalyze distinct steps in strigolactone biosynthesis. Nat. Chem. Biol. 10:1028. doi: $10.1038 /$ nchembio. 1660

Zhang, Y., Zhang, Y., Sun, Q., Lu, S., Chai, L., Ye, J., et al. (2021). Citrus transcription factor CsHB5 regulates abscisic acid biosynthetic genes and promotes senescence. Plant J. 108, 151-168. doi: 10.1111/tpj.15431

Zheng, X., Giuliano, G., and Al-Babili, S. (2020a). Carotenoid biofortification in crop plants: citius, altius, fortius. Biochim. Biophys. Acta Mol. Cell Biol. Lipids 1865:158664. doi: 10.1016/j.bbalip.2020.158664

Zheng, X., Kuijer, H. N., and Al-Babili, S. (2020b). Carotenoid biofortification of crops in the Crispr era. Trends Biotechnol. 39, 857-860. doi: 10.1016/j. tibtech.2020.12.003
Zheng, X., Mi, J., Deng, X., and Al-Babili, S. (2021). LC-MS-based profiling provides new insights into apocarotenoid biosynthesis and modifications in citrus fruits. J. Agric. Food Chem. 69, 1842-1851. doi: 10.1021/acs.jafc.0c06893

Zheng, X., Xie, Z., Zhu, K., Xu, Q., Deng, X., and Pan, Z. (2015). Isolation and characterization of carotenoid cleavage dioxygenase 4 genes from different citrus species. Mol. Gen. Genomics. 290, 1589-1603. doi: 10.1007/ s00438-015-1016-8

Zheng, X., Zhu, K., Sun, Q., Zhang, W., Wang, X., Cao, H., et al. (2019). Natural variation in CCD4 promoter underpins species-specific evolution of red coloration in citrus peel. Mol. Plant 12, 1294-1307. doi: 10.1016/j. molp.2019.04.014

Zhong, Y., Pan, X., Wang, R., Xu, J., Guo, J., Yang, T., et al. (2020). ZmCCD10a encodes a distinct type of carotenoid cleavage dioxygenase and enhances plant tolerance to low phosphate. Plant Physiol. 184, 374-392. doi: 10.1104/ pp. 20.00378

Zhou, X., Welsch, R., Yang, Y., Álvarez, D., Riediger, M., Yuan, H., et al. (2015). Arabidopsis OR proteins are the major posttranscriptional regulators of phytoene synthase in controlling carotenoid biosynthesis. Proc. Natl. Acad. Sci. U. S. A. 112, 3558-3563. doi: 10.1073/pnas.1420831112

Zhu, F., Luo, T., Liu, C., Wang, Y., Yang, H., Yang, W., et al. (2017). An R2R3-MYB transcription factor represses the transformation of $\alpha$-and $\beta$-branch carotenoids by negatively regulating expression of $\mathrm{CrBCH} 2$ and CrNCED5 in flavedo of citrus reticulate. New Phytol. 216, 178-192. doi: $10.1111 /$ nph. 14684

Zhu, F., Luo, T., Liu, C., Wang, Y., Zheng, L., Xiao, X., et al. (2020). A NAC transcription factor and its interaction protein hinder abscisic acid biosynthesis by synergistically repressing NCED5 in Citrus reticulata. J. Exp. Bot. 71, 3613-3625. doi: $10.1093 / \mathrm{jxb} /$ eraa118

Zhu, K., Sun, Q., Chen, H., Mei, X., Lu, S., Ye, J., et al. (2021). Ethylene activation of carotenoid biosynthesis by a novel transcription factor CsERF061. J. Exp. Bot. 72, 3137-3154. doi: 10.1093/jxb/erab047

Zoccali, M., Giuffrida, D., Salafia, F., Rigano, F., Dugo, P., Casale, M., et al. (2021). Apocarotenoids profiling in different capsicum species. Food Chem. 334:127595. doi: 10.1016/j.foodchem.2020.127595

Conflict of Interest: The authors declare that the research was conducted in the absence of any commercial or financial relationships that could be construed as a potential conflict of interest.

The handling editor declared a past co-authorship with one of the authors SA-B.

Publisher's Note: All claims expressed in this article are solely those of the authors and do not necessarily represent those of their affiliated organizations, or those of the publisher, the editors and the reviewers. Any product that may be evaluated in this article, or claim that may be made by its manufacturer, is not guaranteed or endorsed by the publisher.

Copyright (C) 2021 Zheng, Yang and Al-Babili. This is an open-access article distributed under the terms of the Creative Commons Attribution License (CC BY). The use, distribution or reproduction in other forums is permitted, provided the original author(s) and the copyright owner(s) are credited and that the original publication in this journal is cited, in accordance with accepted academic practice. No use, distribution or reproduction is permitted which does not comply with these terms. 